\title{
Análisis de deformación al occidente del Anticlinorio de Los Yariguíes - Cordillera Oriental de Colombia
}

\author{
Lina María Cetina ${ }^{1 *}$, Francisco Velandia ${ }^{1}$, Hugo Alonso Patiño-Sanabria ${ }^{1,2}$
}

doi: http://dx.doi.org/10.18273/revbol.v41n3-2019002 @ ()

Forma de citar: Cetina, L.M., Velandia, F., y Patiño-Sanabria, H.A. (2019). Análisis de deformación al occidente del Anticlinorio de Los Yariguíes - Cordillera Oriental de Colombia. Boletín de Geología, 41(3), 3156. doi: 10.18273/revbol.v41n3-2019002.

Material suplementario electrónico: La versión en línea de este artículo contiene MATERIAL SUPLEMENTARIO (TABLA S1 y S2)

\section{RESUMEN}

El método aplicado en el presente artículo para el análisis de deformación está basado en la evolución de pliegues según inclinación de estratos y en el tratamiento de planos con estrías de falla. El estudio se realizó en el flanco que comparten el Anticlinorio de Los Yariguíes (al oriente) y el Sinclinal de Nuevo Mundo (al occidente), estructuras representativas al NW de la Cordillera Oriental de Colombia. Los datos se sometieron a un análisis mecánico de generación de fracturas según los principios de Anderson y Mohr y se aplicó la técnica de rotación de estratos a los planos con estrías de falla que no cumplieran con presunciones mecánicas. Los resultados del análisis estructural permiten soportar la deformación progresiva que se presenta en la zona con la observación de tres etapas asociadas con distintas condiciones tectónicas. La etapa uno corresponde a distensión-transtensión, con una dirección de máxima extensión de $126^{\circ}$ (NW-SE) asociada al inicio de la acreción de la Cordillera Occidental al final del Mesozoico. La etapa dos se asocia a deformación por compresión-transpresión en el Paleoceno, la cual presenta un tensor de máximo acortamiento en azimut de $30^{\circ}$ (NNE-SSW). Finalmente, la etapa tres corresponde al levantamiento de la Cordillera Oriental en el Oligoceno tardío-Mioceno, asociado a un tensor de acortamiento en dirección $118^{\circ}$ (NWW$\mathrm{SEE}$ ), posiblemente relacionado al inicio de la acreción del bloque Chocó-Panamá. Por otra parte, se establece un estilo estructural con fallas de mayor ángulo que cortan las unidades del Jurásico y de piel delgada que afecta la secuencia cretácica. La zona también está afectada por fallas de rumbo transversales que desplazan levemente las estructuras longitudinales en sentido dextral y dividen el flanco occidental del Anticlinorio de Los Yariguíes, manifestando una deformación diferencial.

Palabras clave: estrías de falla; tensores de deformación; Cordillera Oriental; Valle Medio del Magdalena; Colombia.

\section{Deformation analysis to the west of the Yariguíes Anticlinorium - Eastern Cordillera of Colombia}

\begin{abstract}
The method to carry out the present analysis of deformation is based on the fold evolution according to the strata dip and the treatment of striated fault planes, especially slickensides. We studied the limb that is shared by the Yariguíes Anticlinorium (eastern) and the Nuevo Mundo Syncline (western), both outstanding structures located northwestward of the Eastern Cordillera of Colombia. Fracture data were analyzed under Anderson and Mohr mechanical criteria, and rotation techniques were applied to the rock layers that contain fault planes out of the initial mechanical parameters. The results of the structural analysis support the progressive deformation processes across the area according to three phases that were deduced as related to different tectonic conditions. The first deformation phase consists of distensiontranstension, with an axis of maximum extension toward $126^{\circ}$ (NW-SE), related to accretion onset of the Western Cordillera at the end of the Mesozoic. The second phase is associated with Paleocene compression-transpresion, with a maximum shortening axis to $30^{\circ}$ (NNE-SSW). The third phase corresponds to the onset of uplift of the Eastern Cordillera since the late Oligocene-Miocene, with a shortening tensor to $118^{\circ}$ (NWW-SEE), perhaps related to the accretion beginning of the Chocó-Panamá block. On the other hand, a structural style was defined with higher angles faults that cut the Jurassic units, and thin-skinned for the Cretaceous sequence. Transverse strike-slip faults are also present in the area, cross-cutting the longitudinal structures with dextral displacements, which partitioned the western limb of the Yariguíes Anticlinorium as evidence of differential deformation.
\end{abstract}

Keywords: slickensides; strain tensors; Eastern Cordillera; Middle Magdalena Basin; Colombia.

${ }^{1}$ Escuela de Geología, Universidad Industrial de Santander, Bucaramanga, Colombia. (*) linacetina0526@gmail.com, favelanp@uis.edu.co, hpatino@sgc.gov.co

${ }^{2}$ Servicio Geológico Colombiano, Cali, Colombia. 


\section{INTRODUCCIÓN}

El presente análisis estructural se realiza en un área del piedemonte occidental de la Cordillera Oriental de Colombia, entre el flanco oriental del Sinclinal de Nuevo Mundo (SNM) y occidental del Anticlinorio de Los Yariguíes (AY) (FIGURA 1), mal llamado Anticlinal de Los Cobardes ya que no se trata sólo de un anticlinal (Osorio-Afanador, 2016) y la serranía donde se localiza la estructura hace parte del territorio ancestral de los indígenas Yariguíes o Yareguíes (Velásquez y Castillo, 2006). Esta zona se considera clave para el entendimiento de la evolución geológica de la Cordillera Oriental y de la cuenca del Valle Medio del Magdalena (VMM), una de las principales productoras de hidrocarburos del país.

En los últimos años, diversos estudios efectuados sobre el SNM (e.g. Caballero et al., 2010; Moreno et al., 2011; Nie et al., 2012; Caballero et al., 2013) han establecido varios pulsos de deformación en la zona, asociados a la evolución de la cuenca del VMM. Esto a través de la integración de técnicas multidisciplinarias como medición de paleocorrientes, análisis sedimentológicos, petrografía de areniscas, geocronología $\mathrm{U}-\mathrm{Pb}$ en circones detríticos, entre otros. Sin embargo, no se reporta un estudio estructural que haya sido realizado con base en indicadores cinemáticos obtenidos a partir de deformación frágil, que permitan determinar tensores como explicación para la deformación observada en el área.

En geología estructural se utilizan datos poblacionales de estrías de falla para determinar tensores de presióntensión asociados a la deformación de un cuerpo rocoso (Turner, 1953), o también tensores de esfuerzos a partir de los planos estriados de familias conjugadas (Anderson, 1951; Angelier, 1984; Huang y Angelier, 1989). Las estrías de falla proporcionan una evidencia directa de la deformación frágil que experimentan las rocas $\mathrm{y}$, a su vez, pueden ayudar a determinar la cinemática de las estructuras genéticamente relacionadas con tensores de esfuerzos locales y/o regionales (Angelier, 1984).

El estudio de poblaciones de estrías de fallas ha sido poco aplicado en Colombia, donde se han realizado trabajos a nivel regional en la Cordillera Oriental (Kammer, 1999; Taboada et al., 2000; Velandia, 2017), Valle Superior del Magdalena (Cortés y Angelier, 2005) y otros más localizados en las cordilleras Central y Occidental (López-Cardona, 2006; Mejía et al., 2012) y macizos de Santander y Floresta (Velandia et al., 2016; Velandia y Bermúdez, 2018). El presente trabajo tiene como objetivo principal la obtención de tensores y paleotensores de deformación a partir de ejes de presión-tensión, en un área con relativa continuidad estructural y que presenta un registro estratigráfico completo (desde el Jurásico hasta el Paleógeno), con litologías competentes propicias para el desarrollo de planos estriados, principalmente en unidades del Cretácico (Cetina y Patiño, 2013). Este análisis aporta nuevas evidencias para el entendimiento de la evolución geológica y tectónica del borde noroccidental de la Cordillera Oriental de Colombia. Además, se presenta una actualización de la cartografía geológica existente (Ward et al., 1977) y una propuesta de estilos estructurales para la zona.

\section{MARCO GEOLÓGICO}

\section{Tectónica}

La evolución geológica de los Andes colombianos está relacionada con la interacción de las placas de Nazca, Caribe y Suramericana. Los campos de esfuerzos inducidos por el movimiento de estas placas han generado una intensa deformación, que para la zona de estudio es evidenciada por fallas de cabalgamiento y de rumbo de carácter regional como La Salina, Suárez y Bucaramanga (FIGURA 1), principales estructuras que dominan la geología del área, además del SNM y el AY.

La subducción de la Placa de Nazca provoca actualmente vectores de desplazamiento al NEE (Trenkamp et al., 2002; Mora-Páez et al., 2019), que a su vez inducen un movimiento dextral en las fallas que limitan la Cordillera Oriental al sur de los $4^{\circ}$ latitud norte (Ego et al., 1996), como por ejemplo el Sistema de Fallas de Algeciras al suroeste de Colombia (Velandia et al., 2005). Por otro lado, la interacción con la Placa Caribe ha sido documentada en varios trabajos regionales (e.g. Toto y Kellogg, 1992; Taboada et al., 2000; Trenkamp et al., 2002; Prieto et al., 2012; Bernal-Olaya et al., 2015; Mora-Páez et al., 2019; Restrepo-Moreno et al., 2019) como una gran zona de cizalla al norte de Suramérica, indicando subducción oblicua y subhorizontal (ángulo de 4-8 ${ }^{\circ}$ ) desde el Mioceno medio hasta la actualidad, con una baja tasa de convergencia ( $2 \mathrm{~cm} /$ año) y en dirección WNW-ESE (FIGURA 1). Cabe aclarar aquí que la zona de subducción actual para el Caribe colombiano aún se encuentra en debate, debido principalmente a la falta de arcos magmáticos y a la muy baja sismicidad reportada en el área (e.g. Rossello y Cossey, 2012; Alfaro y Holz, 2014). 


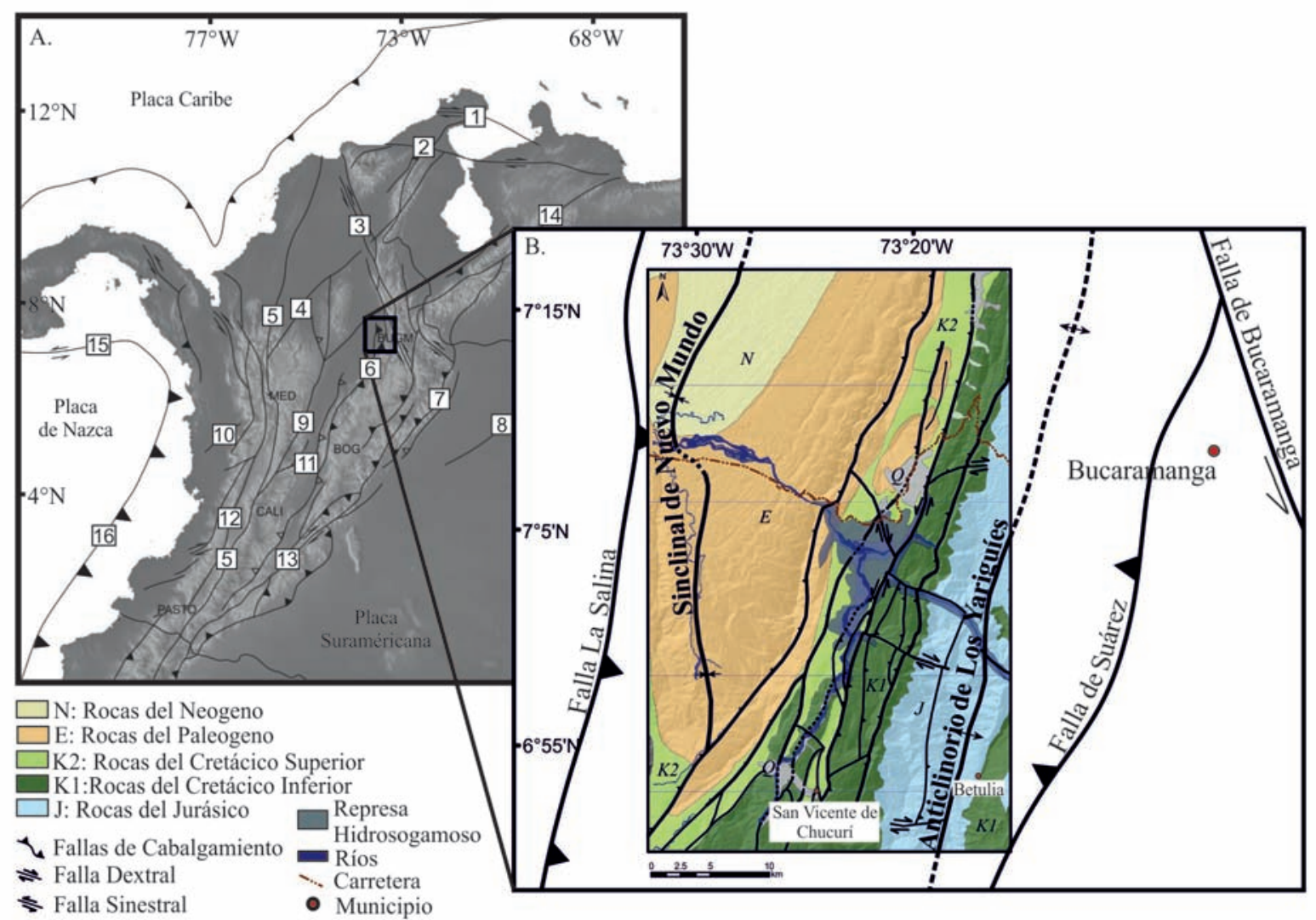

FIGURA 1. A. Esquema tectónico del norte de Suramérica. Modificado de Gómez-Tapias et al. (2007). Abreviaciones: BOG: Bogotá, MED: Medellín, BUGM: Bucaramanga. Fallas: (1) Cuisa; (2) Oca; (3) Santa Marta-Bucaramanga; (4) Espíritu Santo; (5) Cauca-Almaguer; (6) La Salina; (7) Guaicáramo; (8) Meta; (9) Paletina; (10) Zona de Falla Itsmina; (11) Ibagué; (12) Cali-Patía, (13) Algeciras; (14) Boconó; (15) Zona de Falla Transformante Jordán; (16) Zona de Subducción activa Colombo Ecuatoriana. B. Localización del área de estudio y estructuras regionales que afectan la zona. Modificación de Ward et al. (1977).

Esta orientación del tensor de esfuerzos regional (WNW-ESE) se asocia a la generación de un régimen compresivo-transpresivo en las fallas que limitan el borde oriental de la Cordillera Oriental al norte de los $4^{\circ}$ latitud norte (Cediel et al., 2003; Cortés et al., 2005; Tesón et al., 2013). La deformación al norte de esta latitud también se atribuye a la acreción del Bloque Chocó-Panamá contra el flanco NW de la Cordillera Occidental, durante el Oligoceno-Mioceno (DuqueCaro, 1990; Kellogg et al., 1995; Taboada et al., 2000; Farris et al., 2011; Montes et al., 2015; RestrepoMoreno et al., 2019) lo que constituye la causa de la reactivación, inversión y/o generación de la mayor parte de las estructuras que se presentan en la cuenca del VMM y en las estribaciones de la Cordillera Oriental, principalmente en el Mioceno medio. Este régimen transpresivo que afecta los Andes del Norte y genera subsecuente deformación ha sido documentado anteriormente por autores como Taboada et al. (2000), Kammer (1999), Acosta (2002), Cediel et al. (2003), Acosta et al. (2007), Tesón et al. (2013), entre otros.

\section{Pulsos de deformación de Los Andes del Norte en Colombia}

Diversos trabajos en las últimas décadas han propuesto la temporalidad de los pulsos de deformación de los Andes del Norte en Colombia. Los eventos de compresión-transpresión en el territorio colombiano inician con la acreción oblicua de terrenos de afinidad oceánica durante el Cretácico-Paleoceno temprano (e.g. Villagómez et al., 2011; Hincapié-Gómez et al., 2018), lo que contribuyó al inicio de la exhumación de la Cordillera Central, evento marcado en edades de trazas de fisión en circón de 78 Ma (Parra et al., 2009). Posteriormente, la Cordillera Central registra dos eventos de exhumación durante el Eoceno tempranomedio y Oligoceno temprano-Mioceno, así como un evento de calentamiento en el Mioceno tardío-Plioceno tardío, indicado por edades (U-Th)/He en apatito (recopilación en Restrepo-Moreno et al., 2019). Nie et al. (2012) sugieren que el levantamiento inicial de la Cordillera Central se produjo en el Paleoceno medio y 
que se constituyó en fuente de sedimentos importante para la Cuenca del VMM desde el Paleoceno tardío al Eoceno temprano.

Por su parte, la Cordillera Oriental registra la historia de deformación más joven de los tres sistemas montañosos en los Andes colombianos. La mayoría de los autores coinciden en que la formación de la cordillera es producto de una tectónica de inversión sobre una antigua cuenca tipo rifting o back-arc, formada durante el Mesozoico (e.g. McCourt et al., 1984; Cooper et al., 1995; Sarmiento-Rojas et al., 2006; Pindell y Kennan, 2009). Con base en datos de termocronología de baja temperatura, datos sísmicos del VMM e indicadores de paleoesfuerzos en fracturas de pozos, la contracción inicial en el flanco occidental de la presente Cordillera Oriental se presenta en el Paleoceno tardío-Eoceno temprano (Cortés et al., 2005; Parra et al., 2009; Nie et al., 2012; Mora et al., 2013). Por su parte, el levantamiento y acortamiento inicial del eje central de la Cordillera Oriental se daría en el Oligoceno tardío (26-23 Ma) (Villamil, 1999; Horton et al., 2010; Mora et al., 2013; Egbue et al., 2014). Este proceso continuó episódicamente hasta el Mioceno tardío (Cortés et al., 2005; Mora et al., 2013; Velandia, 2017), cuando se registró el máximo levantamiento de la cordillera en respuesta a la colisión del bloque Choco-Panamá (Duque-Caro, 1990; Kellogg et al., 1995; Taboada et al., 2000; Audemard y Audemard, 2002). Finalmente, datos de trazas de fisión en apatito indican un continuo levantamiento tectónico en el Neógeno para la actual Cordillera Oriental (e.g. Parra et al., 2009).

La tectónica de inversión presente en la Cordillera Oriental, según Cortés et al. (2005), dio lugar a una estructura principal tipo pop-up alargada, siguiendo la tendencia de las antiguas fallas normales de la cuenca tipo rifting o back-arc. En ambos flancos de esta estructura se desarrollaron cuencas tipo antepaís (foreland) que acompañaron el levantamiento de la Cordillera Oriental. Así, se proporcionó el espacio de acomodación de sedimentos sin-orogénicos en las cuencas del VMM y Los Llanos Orientales durante el Cenozoico (Cooper et al., 1995; Cortés et al., 2005).

\section{Cuenca del Valle Medio del Magdalena (VMM)}

La cuenca del VMM es una de las más estudiadas en Colombia debido principalmente al interés petrolífero. Investigaciones como las de Ward et al. (1973), Etayo y Rodríguez (1985), Butler y Schamel (1988), Colleta et al. (1990), Dengo y Covey (1993), Cooper et al. (1995),
Sarmiento-Rojas (2001), Royero y Clavijo (2001), Acosta (2002), Gómez et al. (2003, 2005), Mora et al. (2006), Parra et al. (2009), Caballero et al. (2010), Moreno et al. (2011), Nie et al. (2012), Jiménez et al. (2016), entre otros, han permitido importantes avances en el entendimiento de la configuración estructural y estratigráfica de la cuenca.

El VMM es definido como una cuenca hinterland entre las cordilleras Central y Oriental, donde estudios previos han establecido que es el resultado de múltiples fases, incluyendo una extensión tipo rifting o back-arc en el Jurásico-Cretácico Temprano, subsidencia termal en el Cretácico Tardío, e incorporación a una cuenca foreland regional en el Paleógeno, que se fragmentó debido a las etapas de deformación de la Cordillera Oriental en el Cenozoico, con un mecanismo activo de subsidencia flexural (Cooper et al., 1995; Gómez et al., 2005; Sarmiento-Rojas et al., 2006). Estos episodios han tenido registro casi continuo, lo que convierte a la cuenca en un excelente marcador de eventos.

Del Jurásico Tardío al Valanginiano se produce la deposición de las formaciones Girón y Los Santos en un ambiente continental tipo fluvial, mientras que desde el Hauteriviano hasta el Aptiano se deposita la Formación Rosablanca en un ambiente marino somero de plataforma calcárea (Sarmiento-Rojas et al., 2006). Luego, durante el Barremiano se deposita la Formación Paja en un ambiente marino somero (Ward et al., 1973; Sarmiento-Rojas et al., 2006), con secuencias lodosas ricas en fósiles. La acumulación de la secuencia calcárea de la Formación Tablazo ocurre durante el Albiano superior, en un ambiente nerítico poco profundo (Royero y Clavijo, 2001). La Formación Simití se acumula en un ambiente marino de aguas intermedias a profundas, durante el Albiano superior al Cenomaniano (Royero y Clavijo, 2001); en el área de estudio esta unidad se presenta con predominio de lodolitas, con algunas facies calcáreas y abundantes concreciones, incluso de tamaños superiores a un metro de diámetro. La Formación La Luna se deposita en un ambiente marino de aguas poco profundas durante el Turoniano y Santoniano (Morales et al., 1958) y en la zona se caracteriza por calizas muy ricas en fósiles de organismos marinos, con intercalaciones de chert y capas de fosforitas. Sobre esta unidad descansa discordantemente la Formación Umir, del Campaniano-Maastrichtiano (Royero y Clavijo, 2001), que en el área está compuesta principalmente por capas lodosas de color negro. 
Como resultado de la acreción de la Cordillera Occidental se produce un cambio en los ambientes de depósito al comienzo del Cenozoico, pasando de sedimentos marinos del Cretácico a secuencias transicionales con composiciones más clásticas del Paleógeno, en una cuenca de tipo foreland pre-Andina de gran dimensión (e.g. Cooper et al., 1995). La unidad sedimentaria que registra este cambio paleo-ambiental es la Formación Lisama, del Paleoceno, la cual se extendió hacia la cuenca Cesar Ranchería, antes de la separación de las cuencas por efecto de la Falla de Bucaramanga (Campbell, 1968 en Gómez et al., 2005). El cambio de la relativa divergencia a convergencia entre los continentes americanos induce una mudanza importante en las direcciones regionales de máximo esfuerzo, formando la discordancia regional del Eoceno medio en el Valle del Magdalena (Cortés et al., 2005), atribuida también a la máxima deformación de la Cordillera Central (Villamil et al., 1999; Gómez et al., 2003). Así, la Formación La Paz se deposita durante el Eoceno medio y está compuesta principalmente por capas muy potentes de areniscas que superponen en para-conformidad a la Formación Lisama en el flanco oriental del SNM. Posterior a la Formación la Paz ocurre la acumulación de la Formación Esmeraldas desde el Eoceno tardío al Oligoceno temprano, la Formación Mugrosa desde el Oligoceno tardío y la Formación Colorado en el Mioceno temprano-medio (Gómez et al., 2005). Respecto a los grupos Real y Mesa, el primero se deposita desde el Mioceno medio al Mioceno tardío y el segundo durante el Plioceno (Gómez et al., 2005).

\section{MÉTODOS}

Debido a la baja complejidad estructural y a la continuidad estratigráfica del flanco que comparten el SNM y AY, la zona de trabajo presenta condiciones geológicas favorables para la aplicación y evaluación de un método de análisis estructural que permite obtener tensores con ejes de presión y tensión. El área de estudio se caracteriza porque los rumbos de la estratificación se mantienen por varios kilómetros (en promedio 50 $\mathrm{km}$ de longitud, en Ward et al., 1977). Además, en la sucesión de capas competentes e incompetentes la inclinación varía dependiendo de su espesor, ubicación estratigráfica y posición estructural con respecto al SNM y AY. Esta variación en la inclinación actual de las capas sedimentarias (relacionada con su mayor o menor deformación) resulta de gran importancia a la hora de analizar el desarrollo de planos de fallas.
Como parte inicial del análisis estructural en la zona, se detalló la cartografía regional existente (Ward et al., 1977), controlando en campo los contactos y tipos de litologías mediante 73 estaciones (Cetina y Patiño, 2013). Se determinaron pliegues y fallas menores y se definió la vergencia de las estructuras mapeadas (FIGURA 2). Además se elaboraron cortes geológicos y se definieron los estilos estructurales para el área.

Los datos estructurales se completaron con la medición de planos con estrías de fallas en 27 estaciones de campo, con un total de 178 datos distribuidos en las formaciones del Mesozoico y en la Formación Lisama del Paleoceno (FIGURA 2). Además del rumbo y buzamiento de los planos de falla, las estrías fueron medidas según su pitch y dirección de pitch en cuadrantes. Para determinar la cinemática de cada plano de falla se aplicaron criterios de Petit (1987) y Doblas (1998), especialmente indicadores tipo Riedel (R, R') y escalones mineralizados, y se determinó un factor de calidad según la certeza del movimiento y marcación de la estría medida (Velandia, 2017). También se tomó el dato de buzamiento e inclinación de la estratificación de las capas. La TABLA S1 (MATERIAL SUPLEMENTARIO) muestra los datos originales tomados en campo, agrupados por estaciones.

Los datos de estrías de falla fueron recolectados con el objetivo de realizar un análisis de tensores, que se puede asumir desde el punto de vista de esfuerzos o de deformación. La discusión sobre el enfoque más apropiado ha sido tratada en diversos trabajos especializados (Marrett y Peacock, 1999; Fletcher y Pollard, 1999; Peacock y Marrett, 2000), pero en el presente estudio nos acogemos al método de deformación y obtención de ejes de presión y tensión (Marrett y Allmendinger, 1990, 1991) que están relacionados con los ejes de esfuerzos principales mayor y menor, respectivamente, por considerar que reflejan de manera más aproximada el tensor cuando son escasos los planos estriados medidos por estación.

\section{Análisis preliminar de los datos}

Los datos de estrías de falla fueron analizados bajo la premisa de que estos planos pueden reflejar eventos de deformación sobreimpuestos, a pesar de la continuidad estructural del área. Por eso se planteó un método que permite determinar la relación genética que existe entre los planos de falla y la evolución de la deformación del AY, reflejada en la estratificación de las unidades que lo componen (Cetina y Patiño, 2013). Para ello, primero 
se llevó a cabo un análisis puntual preliminar de cada plano de estría de falla, con el objetivo de observar si cada dato cumple con condiciones de generación mecánicamente posibles o sufrió modificaciones por superposición de eventos. El análisis puntual consistió en observar si los datos se acercaban o no las siguientes consideraciones: i) cumplimiento de la teoría de fracturación de Anderson (1951); ii) relaciones angulares ideales entre las estructuras (planos de fallas) según la elipse de deformación, indicando además régimen tectónico propicio; y iii) dato de pitch (o cabeceo de la estría) (TABLA 1). A partir de este análisis se discriminaron de manera preliminar los planos de falla con modificación en su actitud por eventos de deformación sobreimpuestos, a los cuales, posteriormente, se les aplicó la técnica de rotación de planos para identificar sus condiciones ideales de formación.
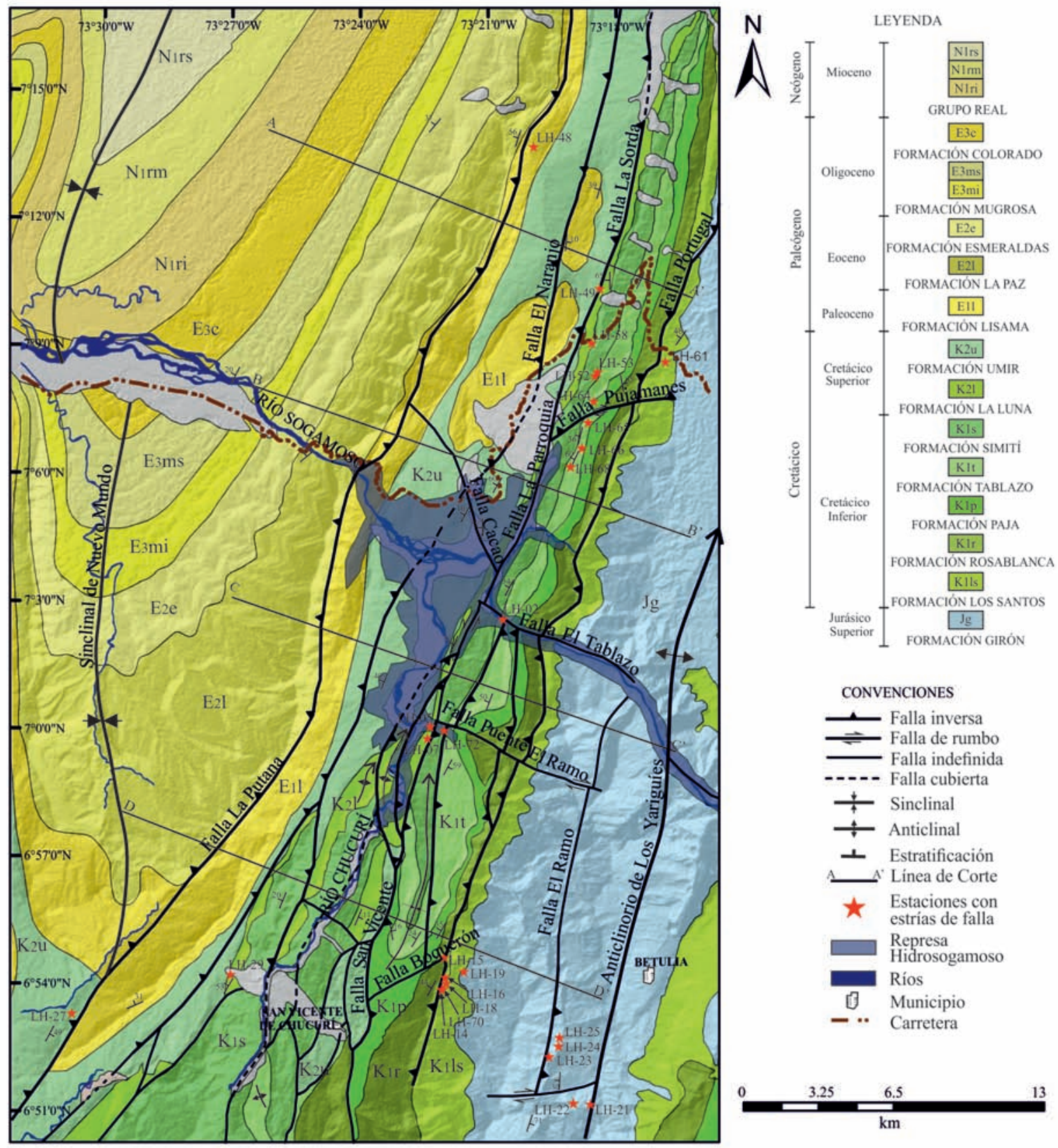

FIGURA 2. Mapa geológico del área de estudio. Modificado de Ward et al. (1977). 
TABLA 1. Características principales de los aspectos considerados para el análisis puntual preliminar (para discriminar datos a rotar).

\begin{tabular}{|c|c|c|}
\hline Consideración & & Características principales \\
\hline \multirow{3}{*}{$\begin{array}{l}\text { Teoría de fracturación de Anderson } \\
\text { (1951) }\end{array}$} & $>$ & Falla normal: ángulo de inclinación $>45^{\circ}$ \\
\hline & $>$ & Falla inversa: ángulo de inclinación $<45^{\circ}$ \\
\hline & $>$ & Falla de rumbo: ángulo de inclinación cercano a la vertical \\
\hline \multirow{3}{*}{$\begin{array}{l}\text { Elipse de deformación } \\
\text { (Régimen tectónico) }\end{array}$} & $>$ & $\begin{array}{l}\text { Fallas normales: rumbo del plano paralelo a eje mayor de deformación. Régimen } \\
\text { Distensivo }\left(\sigma_{1} \text { vertical }\right) \text {. }\end{array}$ \\
\hline & $>$ & $\begin{array}{l}\text { Fallas inversas: rumbo del plano perpendicular al eje mayor de deformación. } \\
\text { Régimen Compresivo }\left(\sigma_{3} \text { vertical). }\right.\end{array}$ \\
\hline & $>$ & $\begin{array}{l}\text { Fallas de rumbo: rumbo del plano oblicuo al eje de mayor deformación. Régimen } \\
\text { Transcurrente }\left(\sigma_{2} \text { vertical). }\right.\end{array}$ \\
\hline \multirow{3}{*}{$\begin{array}{l}\text { Ángulo de pitch (o cabeceo de la } \\
\text { estría) }\end{array}$} & $>$ & Pitch de muy bajo ángulo (<15º): movimiento en el rumbo (fallas de rumbo). \\
\hline & $>$ & $\begin{array}{l}\left.\text { Pitch de muy alto ángulo ( }>75^{\circ}\right) \text { : movimiento en el buzamiento (fallas inversas o } \\
\text { normales). }\end{array}$ \\
\hline & $>$ & Pitch de ángulo intermedio (entre $15^{\circ}$ y $75^{\circ}$ ): movimiento oblicuo. \\
\hline
\end{tabular}

Los datos de estrías de falla que cumplieron con las consideraciones establecidas pasaron directamente al agrupamiento y procesamiento digital, mientras que los datos que se alejaron de estas consideraciones fueron rotados con respecto a un eje horizontal utilizando la plantilla estereográfica de Schmidt. Específicamente, los planos de falla se rotaron con respecto al plano de estratificación de la capa que contiene la fractura, debido a que la inclinación de los estratos se puede relacionar con etapas de formación del pliegue (deformación).

En este procedimiento, la estratificación fue rotada de manera gradual observando el cambio en la actitud y movimiento de los planos de falla hasta que se ajustaran a condiciones óptimas de generación, llevando la estratificación en varios casos a la horizontal (TABLA S2). En la FIGURA 3 se muestra un ejemplo de un dato de estría que fue rotado por no cumplir con las consideraciones asumidas. Este dato corresponde a un plano de falla de movimiento inverso con azimut de rumbo e inclinación $73 / 70$ y pitch de $46^{\circ} \mathrm{E}$ encontrado en una unidad con estratificación de 215/36 (azimut de rumbo/inclinación). El plano de estratificación fue llevado a la horizontal, con un cambio en la actitud del plano de falla a $252 / 80$, del pitch a $68^{\circ} \mathrm{E}$ y del sentido del movimiento del plano a una falla normal dextral. Detalles de la técnica de rotación implementada se puede encontrar en Babín y Gómez (2010) y Cetina y Patiño (2013).
Cabe aclarar que sólo se aplicó la técnica de rotación de planos respecto a un eje horizontal (estratificación), sin considerar rotación con un eje inclinado, que correspondería al cabeceo del anticlinorio, esto por tratarse de una estructura regional que conserva su rumbo por varios kilómetros. Además, la diferencia promedio del rumbo de las capas no es mayor a $15^{\circ} \mathrm{y}$ la mayor variación está asociada a efecto de estructuras locales.

Posteriormente, a partir del grado de rotación efectuado sobre la estratificación de las unidades (en donde están contenidos los planos de falla), se establecieron tres etapas de evolución del AY. Esto debido a que el grado de inclinación de los estratos se relaciona con las etapas de desarrollo del pliegue y con un régimen tectónico asociado. Para ello, se definieron rangos en dos conjuntos según la inclinación actual de las unidades (TABLA 2 y 3 ), ya que se presentan diferencias marcadas en la actitud de los estratos debido a: i) la deformación diferencial que se presenta en la estructura, y ii) a la localización estructural y estratigráfica de cada formación. En ese sentido, estratos con inclinaciones actuales de hasta de $40^{\circ}$ se agruparon en rangos de inclinación de $0^{\circ}$ a $10^{\circ}$, $10^{\circ}$ a $20^{\circ}$, y $20^{\circ}$ a $40^{\circ}$ (TABLA 2), y unidades con inclinaciones actuales mayores a $40^{\circ}$ y hasta $75^{\circ}$ (que corresponde a la máxima inclinación de estratos medidos en campo) se agruparon en rangos de inclinación de $0^{\circ}$ a $10^{\circ}, 10^{\circ}$ a $30^{\circ}$, y $30^{\circ}$ a $75^{\circ}$ (TABLA 3). Estos rangos de inclinación hacen referencia a la estratificación de las 
unidades obtenida después de aplicar la rotación, que para los datos no rotados serán las inclinaciones medidas en campo. De este modo, los estratos que al rotarse quedaron con inclinaciones de $0^{\circ}$ a $10^{\circ}$ se asignaron a una primera etapa (etapa 1), caracterizada por estratos no plegados o levemente basculados asociados a un régimen distensivo; las unidades con inclinaciones de $10^{\circ}$ a $20^{\circ}$ o de $10^{\circ}$ a $30^{\circ}$ (según el caso) se asignaron a una segunda fase (etapa
2), como reflejo de un estadio inicial del plegamiento asociado a un régimen compresivo-transpresivo antiguo; y las unidades con inclinaciones de $20^{\circ}$ a $40^{\circ}$ o de $30^{\circ}$ a $75^{\circ}$ se asignaron a una tercera fase (etapa 3), atribuida a un estadio intermedio-actual del plegamiento y asociado a un régimen compresivo-transpresivo. Cada dato de estría fue agrupado en alguna etapa del plegamiento, según el grado de rotación aplicado (TABLA S2).

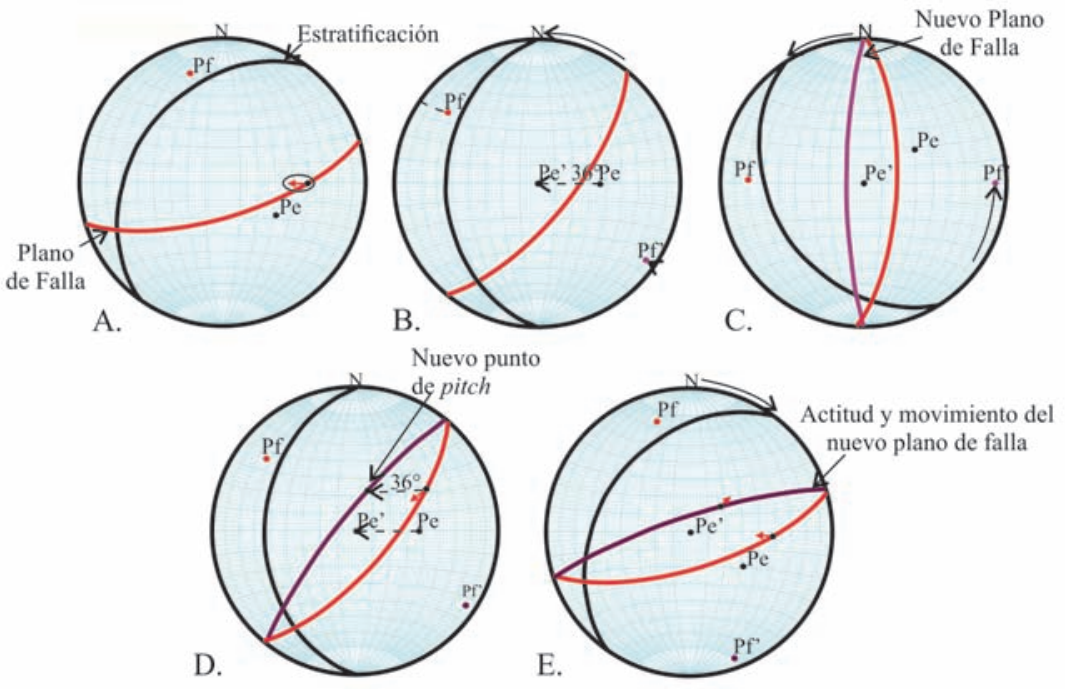

FIGURA 3. Esquema del procedimiento de rotación de plano de falla respecto a la estratificación para una estría de falla inversa (óvalo negro). A. Ubicación del plano de falla (73/70), plano de estratificación (215/36), polo de falla (Pf) y polo de estratificación $(\mathrm{Pe})$. B. Procedimiento inicial de rotación del plano de falla respecto a la estratificación. C. Determinación del nuevo plano de falla. D. Ubicación del pitch en el nuevo plano de falla. E. Nueva actitud y sentido del movimiento del plano de falla una vez rotado respecto a una estratificación horizontal (252/80; pitch $68^{\circ} \mathrm{E}$ y sentido normal dextral).

TABLA 2. Relación de la inclinación de las capas con respecto a una etapa de desarrollo del pliegue y el tipo de régimen tectónico asociado. Se incluyen las unidades con inclinación actual hasta $40^{\circ}$.

\begin{tabular}{cccc}
\hline $\begin{array}{c}\text { Rango de inclinación } \\
\text { de unidades }\end{array}$ & Desarrollo del plegamiento & Régimen asociado & Etapas \\
\hline $0^{\circ}-10^{\circ}$ & Unidades no plegadas & Régimen distensivo & 1 \\
$10^{\circ}-20^{\circ}$ & Estadio inicial del plegamiento & Régimen compresivo/transpresivo antiguo & 2 \\
$20^{\circ}-40^{\circ}$ & Estadio intermedio o actual del & Régimen compresivo/transpresivo & 3 \\
& plegamiento & intermedio o actual & 3 \\
\hline
\end{tabular}

TABLA 3. Relación de la inclinación de las capas con respecto a una etapa de desarrollo del pliegue y el tipo de régimen tectónico asociado. Se incluyen las unidades con inclinación actual de $40^{\circ}$ a $75^{\circ}$ (máxima inclinación medida en el área).

\begin{tabular}{cccc}
\hline $\begin{array}{c}\text { Rango de inclinación } \\
\text { de unidades }\end{array}$ & Desarrollo del plegamiento & Régimen asociado & Etapas \\
\hline $0^{\circ}-10^{\circ}$ & Unidades no plegadas & Régimen distensivo & 1 \\
$10^{\circ}-30^{\circ}$ & Estadio inicial del plegamiento & Régimen compresivo/transpresivo \\
& antiguo & 2 \\
$30^{\circ}-75^{\circ}$ & Estadio intermedio o actual del & plegamiento & $\begin{array}{c}\text { Régimen compresivo/transpresivo } \\
\text { intermedio o actual }\end{array}$ \\
\hline
\end{tabular}


Los datos que a pesar de ser rotados no mostraron coherencia mecánica (cumplimiento de los principios de fracturación de Anderson, elipse de deformación y ángulo de pitch, TABLA 1) fueron eliminados, considerando además la calidad del dato registrado en campo. Lo anterior constituyó un primer filtro para los datos recolectados en campo.
En la TABLA 4 se observa el resultado del análisis puntual preliminar de los datos correspondientes a la estación LH18 y en la TABLA S2 se muestra para las demás estaciones con datos de estrías. Cabe aclarar que los datos de pitch fueron convertidos a datos de inmersión y dirección de inmersión (plunge y trend) utilizando la plantilla estereográfica de Schmidt (Cetina y Patiño, 2013).

TABLA 4. Resultado del análisis y tratamiento previo de los datos en la estación LH-18.

\begin{tabular}{|c|c|c|c|c|c|c|c|c|}
\hline $\begin{array}{c}\text { Estación/ } \\
\text { Estratificación }\end{array}$ & No. & $\begin{array}{c}\text { Azimut de } \\
\text { Buzamiento }\end{array}$ & Inclinación & $\begin{array}{c}\text { Dir. } \\
\text { Inmersión } \\
(\text { Trend })\end{array}$ & $\begin{array}{c}\text { Inmersión } \\
\text { (Plunge) }\end{array}$ & *Sentido & $\begin{array}{c}\text { Nueva } \\
\text { inclinación de } \\
\text { Estratificación }\end{array}$ & $\begin{array}{l}\text { Ángulo de } \\
\text { Rotación }\end{array}$ \\
\hline \multirow{12}{*}{ LH-18 } & 1 & 253 & 41 & 182 & 15 & 4 & $43^{\circ}$ & $0^{\circ}$ \\
\hline & 2 & 278 & 73 & 335 & 60 & 2 & $10^{\circ}$ & $33^{\circ}$ \\
\hline & 3 & 298 & 88 & 24 & 55 & 2 & $10^{\circ}$ & $33^{\circ}$ \\
\hline & 4 & 68 & 55 & 341 & 3 & 3 & $20^{\circ}$ & $13^{\circ}$ \\
\hline & 5 & 298 & 58 & 311 & 58 & 2 & \multicolumn{2}{|c|}{ eliminado } \\
\hline & 6 & 321 & 55 & 9 & 46 & 2 & \multicolumn{2}{|c|}{ eliminado } \\
\hline & 7 & 208 & 35 & 151 & 21 & 3 & $43^{\circ}$ & $0^{\circ}$ \\
\hline & 8 & 308 & 76 & 357 & 68 & 2 & $10^{\circ}$ & $33^{\circ}$ \\
\hline & 9 & 259 & 36 & 261 & 35 & 1 & $43^{\circ}$ & $0^{\circ}$ \\
\hline & 10 & 248 & 35 & 267 & 33 & 2 & \multicolumn{2}{|c|}{ eliminado } \\
\hline & 11 & 293 & 44 & 258 & 38 & 2 & \multicolumn{2}{|c|}{ eliminado } \\
\hline & 12 & 278 & 43 & 283 & 43 & 1 & $43^{\circ}$ & $0^{\circ}$ \\
\hline \multirow{9}{*}{$210 / 43$} & $\begin{array}{l}\text { Nuevo } \\
\text { No }\end{array}$ & $\begin{array}{c}\text { Nuevo } \\
\text { Azimut de } \\
\text { Buzamiento }\end{array}$ & $\begin{array}{c}\text { Nueva } \\
\text { Inclinación }\end{array}$ & $\begin{array}{l}\text { Nueva } \\
\text { Dir. } \\
\text { Inmersión } \\
\text { (Trend) }\end{array}$ & $\begin{array}{c}\text { Nueva } \\
\text { Inmersión } \\
\text { (Plunge) }\end{array}$ & $\begin{array}{l}\text { Nuevo } \\
\text { sentido }\end{array}$ & \multicolumn{2}{|c|}{$\begin{array}{c}* * \text { Etapa de } \\
\text { desarrollo del } \\
\text { pliegue }\end{array}$} \\
\hline & 1 & 253 & 41 & 182 & 15 & 4 & \multicolumn{2}{|c|}{3} \\
\hline & 2 & 269 & 45 & 321 & 31 & 2 & \multicolumn{2}{|c|}{1} \\
\hline & 3 & 298 & 55 & 348 & 30 & 2 & \multicolumn{2}{|c|}{1} \\
\hline & 4 & 77 & 71 & 162 & 13 & 3 & \multicolumn{2}{|c|}{2} \\
\hline & 5 & 208 & 35 & 151 & 21 & 3 & \multicolumn{2}{|c|}{3} \\
\hline & 6 & 311 & 45 & 325 & 42 & 2 & \multicolumn{2}{|c|}{1} \\
\hline & 7 & 259 & 36 & 261 & 35 & 1 & \multicolumn{2}{|c|}{3} \\
\hline & 8 & 278 & 43 & 283 & 43 & 1 & \multicolumn{2}{|c|}{3} \\
\hline
\end{tabular}

* Código de movimiento, 1: F. inversa; 2: F. normal; 3: F. dextral; 4: F. sinestral

**Código de Etapa de Plegamiento, 1:Etapa uno; 2: Etapa dos; 3: Etapa tres

\section{Procesamiento de los datos de estrías de falla}

El procesamiento de los datos de estrías se realizó utilizando el software TectonicsFP (Reiter y Acs, 19962014), especializado en el manejo y procesamiento de datos estructurales. Con el objetivo de comprobar la superposición de eventos se generaron inicialmente gráficos de Angelier o proyección estereográfica de los planos y estrías de falla (Angelier, 1984) y Círculo de Mohr para los datos agrupados según su localización (por estaciones individuales o grupos de estaciones muy cercanas entre sí). El gráfico de
Angelier fue empleado para mostrar la orientación de los planos de falla por estación (ejemplo estación LH18, FIGURA 4A) y el Circulo de Mohr para analizar la coherencia mecánica de los planos según su ubicación en el dominio inestable, de reactivación o estable (Martínez, 2002; Fossen, 2010; Burg, 2011; FIGURA 4B). Con base en estos gráficos se observó si los datos efectivamente correspondían o no a un mismo tensor de deformación (ejes de presión-tensión) y, a su vez, se usó como criterio para eliminar los datos del dominio estable, aplicando así un segundo filtro. 
Demostrada la superposición de eventos en las estaciones de campo y realizada la rotación de los planos según el análisis puntual preliminar, se agruparon los datos según las etapas de desarrollo del pliegue, es decir, a las etapas uno, dos y tres del plegamiento (TABLAS 2, 3, 4 y S2). Una vez discriminados los datos por etapas de formación del pliegue, se generaron gráficos de diedros rectos NDA (numerical dynamic analysis) y P-T (ejes de presión- tensión) para determinar los paleotensores o tensores actuales responsables de la deformación (FIGURA 5). Por su parte, la cinemática de las fallas se determinó con base en el tensor obtenido al procesar los datos correspondientes a la etapa más reciente de la deformación (etapa tres).

El proceso descrito para cada una de las estaciones, se efectuó también agrupando los datos por formaciones geológicas. Esto con el propósito de analizar las variaciones de los tensores (o paleotensores) a través del tiempo geológico. Como ejemplo, en la TABLA 5 se muestra el resultado de rotación de los datos correspondientes a la Formación La Luna y su agrupación según las etapas del pliegue establecidas.

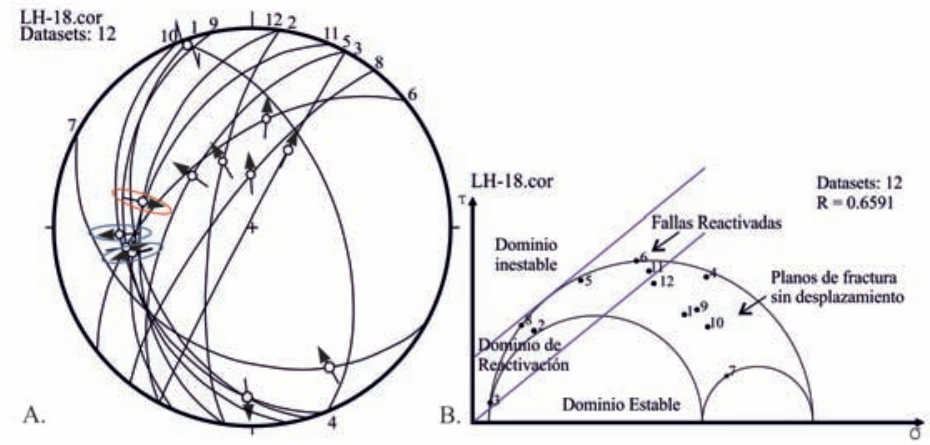

FIGURA 4. Análisis mecánico de planos con estrías de falla. A. Gráfico de Angelier correspondiente a la estación LH-18, donde se observan fallas normales (óvalo azul) aproximadamente paralelas a una falla inversa (óvalo rojo), incompatibles si se ubican en una misma elipse de deformación por corresponder a distintos campos de esfuerzos. B. Círculo de Mohr para los datos de la estación LH-18, donde se grafican los tres dominios para observar las poblaciones de fallas que coexisten en un mismo afloramiento, pero se generaron por diversos campos de esfuerzos.

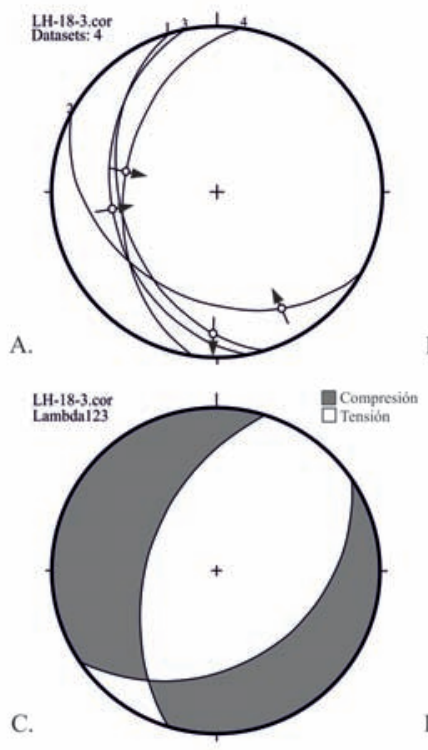

B.
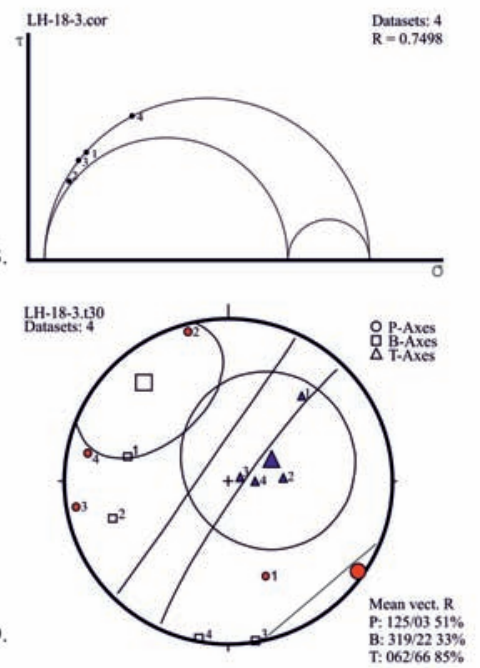

FIGURA 5. Diagramas obtenidos en el procesamiento de los datos agrupados en la etapa tres de la estación LH-18 (ver TABLA 4). A. Gráfico de Angelier, donde se observa la orientación de los planos y ubicación de la estría de falla. B. Círculo de Mohr, que muestra los datos en el dominio inestable, es decir, generados por un mimo tensor. C. Gráfico de diedros rectos NDA, el cual refleja el régimen compresivo en que se formaron los planos. D. Gráfico P-T, donde se muestran las direcciones de presión y tensión propicias para la generación de las fallas. 
Finalmente, los paleotensores y tensores de deformación, para cada una de las etapas de desarrollo del pliegue con ejes de presión-tensión, se obtuvieron mediante el (etapa uno, dos y tres) con ayuda de gráficos de Angelier, procesamiento de los datos de estrías de falla agrupados Círculo de Mohr, diagramas NDA y ejes P-T.

TABLA 5. Análisis puntual preliminar para datos de la Formación La Luna.

\begin{tabular}{|c|c|c|c|c|c|c|c|c|}
\hline Estación & Estrati. & $\begin{array}{l}\text { Azimut de } \\
\text { Buzamiento }\end{array}$ & Inclinación & $\begin{array}{c}\text { Dir. } \\
\text { Inmersión } \\
\text { (Trend) }\end{array}$ & $\begin{array}{c}\text { Inmersión } \\
\text { (Plunge) }\end{array}$ & *Sentido & $\begin{array}{c}\text { Ángulo de } \\
\text { rotación }\end{array}$ & \\
\hline \multirow[b]{2}{*}{ LH-05 } & \multirow[b]{2}{*}{$20 / 55$} & 195 & 70 & 117 & 30 & 3 & $0^{\circ}$ & \\
\hline & & 197 & 79 & 118 & 44 & 3 & $25^{\circ}$ & \\
\hline \multirow{2}{*}{ LH-07 } & \multirow{2}{*}{$36 / 62$} & 43 & 74 & 130 & 3 & 3 & $0^{\circ}$ & \\
\hline & & 53 & 79 & 341 & 58 & 2 & $0^{\circ}$ & \\
\hline LH-29 & $210 / 53$ & 295 & 53 & 305 & 52 & 1 & $0^{\circ}$ & \\
\hline \multirow{8}{*}{ LH-49 } & \multirow{8}{*}{$180 / 65$} & 265 & 65 & 277 & 64 & 1 & $45^{\circ}$ & \\
\hline & & 100 & 55 & 104 & 54 & 1 & $65^{\circ}$ & \\
\hline & & 285 & 61 & 270 & 60 & 1 & $45^{\circ}$ & \\
\hline & & 321 & 76 & 259 & 62 & 1 & $0^{\circ}$ & \\
\hline & & 308 & 45 & 298 & 45 & 1 & $0^{\circ}$ & \\
\hline & & 319 & 60 & 282 & 54 & 1 & $20^{\circ}$ & \\
\hline & & 325 & 68 & 280 & 58 & 1 & $0^{\circ}$ & \\
\hline & & 298 & 43 & 294 & 42 & 1 & $0^{\circ}$ & \\
\hline \multirow{16}{*}{ LH-058 } & \multirow{16}{*}{$184 / 56$} & 200 & 30 & 238 & 24 & 1 & $46^{\circ}$ & \\
\hline & & 220 & 33 & 223 & 33 & 1 & $46^{\circ}$ & \\
\hline & & 204 & 39 & 223 & 38 & 1 & $46^{\circ}$ & \\
\hline & & 210 & 30 & 216 & 30 & 1 & $46^{\circ}$ & \\
\hline & & 110 & 70 & 124 & 68 & 1 & $56^{\circ}$ & \\
\hline & & 106 & 68 & 67 & 62 & 1 & $56^{\circ}$ & \\
\hline & & 110 & 62 & 120 & 61 & 1 & $56^{\circ}$ & \\
\hline & & 108 & 70 & 54 & 57 & 1 & $56^{\circ}$ & \\
\hline & & 180 & 65 & 192 & 65 & 2 & $0^{\circ}$ & \\
\hline & & 165 & 60 & 176 & 59 & 2 & $46^{\circ}$ & \\
\hline & & 190 & 56 & 196 & 58 & 1 & $46^{\circ}$ & \\
\hline & & 195 & 62 & 200 & 62 & 1 & $46^{\circ}$ & \\
\hline & & 175 & 60 & 203 & 58 & 1 & $46^{\circ}$ & \\
\hline & & 214 & 84 & 302 & 24 & 4 & $0^{\circ}$ & \\
\hline & & 300 & 24 & 311 & 23 & 1 & $0^{\circ}$ & \\
\hline & & 325 & 22 & 336 & 21 & 1 & $0^{\circ}$ & \\
\hline Estación & $\begin{array}{c}\text { Nueva } \\
\text { inclinación } \\
\text { de Estrati. }\end{array}$ & $\begin{array}{c}\text { Nuevo } \\
\text { Azimut de } \\
\text { Buzamiento }\end{array}$ & $\begin{array}{c}\text { Nueva } \\
\text { Inclinación }\end{array}$ & $\begin{array}{c}\text { NuevaDir. } \\
\text { Inmersión } \\
\text { (Trend) }\end{array}$ & $\begin{array}{c}\text { Nueva } \\
\text { Inmersión } \\
\text { (Plunge) }\end{array}$ & $\begin{array}{l}\text { Nuevo } \\
\text { sentido }\end{array}$ & Calidad & $\begin{array}{c}* * \text { Etapa de } \\
\text { Plega- } \\
\text { miento }\end{array}$ \\
\hline \multirow{2}{*}{ LH-05 } & $55^{\circ}$ & 195 & 70 & 117 & 30 & 3 & 2 & 3 \\
\hline & $30^{\circ}$ & 197 & 79 & 118 & 44 & 3 & 2 & 3 \\
\hline \multirow{2}{*}{ LH-07 } & $62^{\circ}$ & 43 & 74 & 130 & 3 & 3 & 2 & 3 \\
\hline & $62^{\circ}$ & 53 & 79 & 341 & 58 & 2 & 3 & 3 \\
\hline LH-29 & $53^{\circ}$ & 295 & 50 & 305 & 49 & 1 & 3 & 3 \\
\hline \multirow{8}{*}{ LH-49 } & $20^{\circ}$ & 256 & 20 & 274 & 20 & 1 & 2 & 2 \\
\hline & $0^{\circ}$ & 280 & 60 & 256 & 59 & 2 & 2 & 1 \\
\hline & $20^{\circ}$ & 311 & 20 & 270 & 15 & 1 & 2 & 2 \\
\hline & $65^{\circ}$ & 321 & 76 & 259 & 62 & 1 & 1 & 3 \\
\hline & $65^{\circ}$ & 308 & 45 & 298 & 45 & 1 & 1 & 3 \\
\hline & $45^{\circ}$ & 330 & 46 & 281 & 32 & 1 & 1 & 3 \\
\hline & $65^{\circ}$ & 325 & 68 & 280 & 58 & 1 & 1 & 3 \\
\hline & $65^{\circ}$ & 298 & 43 & 294 & 42 & 1 & 2 & 3 \\
\hline \multirow{16}{*}{ LH-058 } & $10^{\circ}$ & 124 & 68 & 53 & 39 & 1 & 1 & 2 \\
\hline & $10^{\circ}$ & 126 & 58 & 47 & 16 & 4 & 1 & 2 \\
\hline & $10^{\circ}$ & 134 & 65 & 54 & 18 & 4 & 1 & 2 \\
\hline & $10^{\circ}$ & 124 & 63 & 43 & 17 & 4 & 1 & 2 \\
\hline & $0^{\circ}$ & 299 & 40 & 262 & 33 & 2 & 3 & 1 \\
\hline & $0^{\circ}$ & 294 & 40 & 291 & 40 & 2 & 3 & 1 \\
\hline & $0^{\circ}$ & 294 & 45 & 257 & 39 & 2 & 3 & 1 \\
\hline & $0^{\circ}$ & 297 & 39 & 300 & 39 & 2 & 3 & 1 \\
\hline & $56^{\circ}$ & 180 & 65 & 192 & 65 & 2 & 2 & 3 \\
\hline & $10^{\circ}$ & 328 & 82 & 241 & 24 & 4 & 2 & 1 \\
\hline & $10^{\circ}$ & 151 & 76 & 241 & 4 & 4 & 2 & 1 \\
\hline & $10^{\circ}$ & 159 & 73 & 247 & 8 & 4 & 2 & 1 \\
\hline & $10^{\circ}$ & 155 & 89 & 245 & 1 & 4 & 2 & 1 \\
\hline & $56^{\circ}$ & 214 & 84 & 302 & 24 & 4 & 2 & 3 \\
\hline & $56^{\circ}$ & 300 & 24 & 311 & 23 & 1 & 2 & 3 \\
\hline & $56^{\circ}$ & 325 & 22 & 336 & 21 & 1 & 3 & 3 \\
\hline
\end{tabular}

* Código de movimiento, 1: F. inversa; 2: F. normal; 3: F. dextral; 4: F. sinestral

**Código de Etapa de Plegamiento, 1:Etapa uno; 2: Etapa dos; 3: Etapa tres 


\section{RESULTADOS}

En primer lugar se muestran los resultados de los tensores por formaciones geológicas y estaciones, indicando la orientación de los ejes de Presión (P), Intermedio (B) y Tensión ( $\mathrm{T}$ ) y la correspondencia con las etapas del desarrollo del pliegue hacia el flanco occidental del AY (TABLA 6). También se indica el régimen tectónico asociado, que se define por el tipo de gráfico de diedros rectos que arroja el método NDA y por las orientaciones de los ejes P, B y T, considerando especialmente la posición del eje vertical, donde $\mathrm{P}$ cerca a la vertical indica régimen distensivo, $\mathrm{B}$ régimen transcurrente y T régimen compresivo.

TABLA 6. Tensores de deformación asociados a las etapas de desarrollo del flanco occidental del AY.

\begin{tabular}{|c|c|c|c|c|c|c|c|c|c|c|}
\hline Formación & Estación & $\begin{array}{c}\text { Estrati. } \\
\text { Az. } \\
\text { Rumbo } \\
\end{array}$ & $\begin{array}{c}\text { Ángulo } \\
\text { de } \\
\text { rotación }\end{array}$ & $\begin{array}{c}\text { Nueva } \\
\text { inclinación } \\
\text { de Estrat. }\end{array}$ & $\begin{array}{c}\text { Etapa de } \\
\text { Plegamiento }\end{array}$ & (P) & (B) & (T) & $\begin{array}{c}\text { Número } \\
\text { de estrías } \\
\text { de falla }\end{array}$ & $\begin{array}{l}\text { Régimen } \\
\text { Tectónico }\end{array}$ \\
\hline \multirow{6}{*}{$\begin{array}{l}\text { La Luna } \\
\text { K2l }\end{array}$} & \multirow{3}{*}{ LH-49 } & \multirow{3}{*}{$180 / 65$} & $65^{\circ}$ & $0^{\circ}$ & 1 & $189 / 80$ & $004 / 9$ & $094 / 01$ & 1 & Distensivo \\
\hline & & & $45^{\circ}$ & $20^{\circ}$ & 2 & 095/11 & $005 / 4$ & $247 / 74$ & 2 & Compresivo \\
\hline & & & $20^{\circ}-0^{\circ}$ & $45^{\circ}-65^{\circ}$ & 3 & $298 / 22$ & $036 / 16$ & $160 / 62$ & 5 & Compresivo \\
\hline & \multirow{3}{*}{ LH-058 } & \multirow{3}{*}{$184 / 56$} & $56^{\circ}-46^{\circ}$ & $0^{\circ}-10^{\circ}$ & 1 & $225 / 39$ & $025 / 48$ & $116 / 05$ & 8 & Transtensivo \\
\hline & & & $46^{\circ}$ & $10^{\circ}$ & 2 & $033 / 30$ & $176 / 54$ & 289/123 & 4 & Transpresivo \\
\hline & & & $0^{\circ}$ & $56^{\circ}$ & 3 & $306 / 21$ & $073 / 16$ & $002 / 44$ & 4 & Transpresivo \\
\hline $\begin{array}{l}\text { Simití } \\
\text { K1s }\end{array}$ & LH-68 & $010 / 25$ & $0^{\circ}$ & $25^{\circ}$ & 3 & $125 / 17$ & $298 / 73$ & $035 / 02$ & 5 & Transcurrente \\
\hline \multirow{6}{*}{$\begin{array}{c}\text { Tablazo } \\
\text { K1t }\end{array}$} & LH-02 & $210 / 34$ & $34^{\circ}$ & $0^{\circ}$ & 1 & $212 / 61$ & $092 / 15$ & $345 / 24$ & 4 & Distensivo \\
\hline & \multirow{3}{*}{ LH-52 } & \multirow{3}{*}{$200 / 56$} & $56^{\circ}$ & $0^{\circ}$ & 1 & $252 / 88$ & $264 / 4$ & $354 / 3$ & 1 & Distensivo \\
\hline & & & $31^{\circ}$ & $25^{\circ}$ & 2 & $114 / 4$ & $210 / 80$ & $025 / 9$ & 1 & Transcurrente \\
\hline & & & $0^{\circ}$ & $56^{\circ}$ & 3 & $111 / 30$ & $276 / 59$ & $017 / 07$ & 4 & Transcurrente \\
\hline & \multirow{2}{*}{ LH-53 } & \multirow{2}{*}{$205 / 60$} & $50^{\circ}$ & $10^{\circ}$ & 2 & $201 / 20$ & $293 / 03$ & $033 / 70$ & 5 & Compresivo \\
\hline & & & $0^{\circ}$ & $60^{\circ}$ & 3 & 098/52 & $269 / 37$ & $002 / 03$ & 5 & Transtensivo \\
\hline \multirow{7}{*}{$\begin{array}{c}\text { Tablazo } \\
\text { K1t }\end{array}$} & *LH-64 & $004 / 30$ & $30^{\circ}-0^{\circ}$ & $0^{\circ}-30^{\circ}$ & 3 & $123 / 16$ & $278 / 73$ & $031 / 06$ & 9 & Transcurrente \\
\hline & \multirow{2}{*}{ LH-65 } & \multirow{2}{*}{$200 / 50$} & $40^{\circ}$ & $10^{\circ}$ & 2 & $049 / 03$ & $114 / 58$ & $155 / 20$ & 3 & Transpresivo \\
\hline & & & $20^{\circ}-0^{\circ}$ & $30^{\circ}-50^{\circ}$ & 3 & $130 / 05$ & $254 / 29$ & $059 / 45$ & 8 & Transpresivo \\
\hline & LH-66 & $205 / 60$ & $0^{\circ}$ & $60^{\circ}$ & 1 & $290 / 16$ & $078 / 68$ & $197 / 7$ & 2 & Transcurrente \\
\hline & \multirow{3}{*}{ LH-72 } & \multirow{3}{*}{$200 / 50$} & $50^{\circ}$ & $0^{\circ}$ & 1 & $328 / 4$ & $230 / 72$ & $060 / 17$ & 1 & Transcurrente \\
\hline & & & $40^{\circ}$ & $10^{\circ}$ & 2 & $176 / 20$ & $310 / 63$ & 080/17 & 1 & Transcurrente \\
\hline & & & $0^{\circ}$ & $50^{\circ}$ & 3 & $138 / 05$ & $186 / 49$ & 073/38 & 11 & Transpresivo \\
\hline \multirow{9}{*}{$\begin{array}{c}\text { Rosablanca } \\
\text { K1r }\end{array}$} & \multirow{2}{*}{ LH-14 } & \multirow{2}{*}{$260 / 17$} & $17^{\circ}$ & $0^{\circ}$ & 1 & $054 / 73$ & $199 / 13$ & $291 / 09$ & 5 & Distensivo \\
\hline & & & $0^{\circ}$ & $17^{\circ}$ & 3 & 298/18 & $034 / 19$ & $168 / 65$ & 1 & Compresivo \\
\hline & \multirow{3}{*}{ LH-15 } & \multirow{3}{*}{$215 / 36$} & $36^{\circ}-26^{\circ}$ & $0^{\circ}-10^{\circ}$ & 1 & $052 / 59$ & $256 / 29$ & $159 / 07$ & 4 & Transtensivo \\
\hline & & & $26^{\circ}$ & $10^{\circ}$ & 2 & $338 / 24$ & $086 / 12$ & $168 / 53$ & 3 & Compresivo \\
\hline & & & $0^{\circ}$ & $36^{\circ}$ & 3 & $317 / 07$ & $239 / 03$ & 020/59 & 4 & Transpresivo \\
\hline & LH-16 & $215 / 36$ & $26^{\circ}$ & $10^{\circ}$ & 1 & $267 / 68$ & $005 / 2$ & $094 / 20$ & 1 & Distensivo \\
\hline & \multirow{3}{*}{ LH-18 } & \multirow{3}{*}{$210 / 43$} & $33^{\circ}$ & $10^{\circ}$ & 1 & $005 / 62$ & $225 / 23$ & $128 / 116$ & 3 & Distensivo \\
\hline & & & $23^{\circ}$ & $20^{\circ}$ & 2 & $190 / 23$ & $44 / 65$ & $285 / 10$ & 1 & Transtensivo \\
\hline & & & $0^{\circ}$ & $43^{\circ}$ & 3 & $125 / 03$ & $319 / 22$ & 062/66 & 4 & Compresivo \\
\hline \multirow{3}{*}{$\begin{array}{c}\text { Los Santos } \\
\text { K1ls }\end{array}$} & LH-61 & $214 / 48$ & $0^{\circ}$ & $48^{\circ}$ & 3 & $287 / 20$ & $020 / 07$ & $129 / 69$ & 4 & Compresivo \\
\hline & 7 & $185 / 30$ & $20^{\circ}$ & $10^{\circ}$ & 2 & $024 / 12$ & $147 / 66$ & $290 / 18$ & 1 & Transcurrente \\
\hline & LH-70 & $185 / 30$ & $0^{\circ}$ & $30^{\circ}$ & 3 & $344 / 08$ & $263 / 32$ & 092/55 & 4 & Compresivo \\
\hline & LH-19 & $190 / 34$ & $0^{\circ}$ & $34^{\circ}$ & 3 & $232 / 78$ & $262 / 6$ & $185 / 5$ & 2 & Distensivo \\
\hline & LH-21 & $185 / 53$ & $0^{\circ}$ & $53^{\circ}$ & 3 & $285 / 19$ & $148 / 29$ & $112 / 50$ & 2 & Compresivo \\
\hline & LH-22 & $275 / 15$ & $0^{\circ}$ & $15^{\circ}$ & 3 & $121 / 10$ & $284 / 79$ & $030 / 03$ & 4 & Transcurrente \\
\hline & LH-23 & $285 / 20$ & $0^{\circ}$ & $20^{\circ}$ & 3 & $283 / 24$ & $124 / 63$ & $019 / 10$ & 2 & Transpresivo \\
\hline Girón & 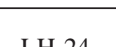 & $032 / 65$ & $55^{\circ}$ & $10^{\circ}$ & 2 & $006 / 7$ & $96 / 18$ & $254 / 68$ & 1 & Compresivo \\
\hline & LH-24 & 032/65 & $35^{\circ}-0^{\circ}$ & $30^{\circ}-65^{\circ}$ & 3 & $120 / 05$ & 029/08 & $263 / 80$ & 9 & Compresivo \\
\hline & & & $45^{\circ}$ & $0^{\circ}$ & 1 & $137 / 75$ & $248 / 5$ & $340 / 16$ & 2 & Distensivo \\
\hline & LH-25 & $020 / 45$ & $35^{\circ}-15^{\circ}$ & $10^{\circ}-30^{\circ}$ & 2 & $187 / 12$ & $256 / 20$ & $331 / 45$ & 3 & Compresivo \\
\hline & & & $10^{\circ}$ & $35^{\circ}$ & 3 & $149 / 4$ & $59 / 19$ & $244 / 70$ & 2 & Compresivo \\
\hline
\end{tabular}

\footnotetext{
* En la estación LH-64 se presentan replegamientos en el flanco oeste del AY, los cuales debieron ser originados en una etapa intermedia a tardía del desarrollo del pliegue, por lo que rotar a cero la estratificación en este caso no significa restaurar la estratificación a un estadio depositacional pre deformativo.
} 
Los planos medidos (y agrupados en la etapa 3) se muestran mediante gráficos de Angelier para cada una de las estaciones de trabajo que presentan por lo menos cuatro datos de estrías de falla (FIGURA 6). También se muestran los tensores correspondientes en forma de balones de playa según diedros rectos NDA para cada estación (FIGURA 6). Con estos gráficos NDA se identifica el rumbo y buzamiento de las fallas que el programa propone como solución, las estructuras principales y asociadas, así como la orientación de los ejes de presión-tensión que explican la cinemática de las fallas involucradas. Las fallas definidas para el área de estudio (FIGURAS 2 y 6) corresponden al procesamiento de los datos de estrías en la etapa tres. Este conjunto de datos se utiliza porque corresponde a la última etapa de desarrollo de los pliegues y fallas como resultado de la deformación finita y progresiva. El control y detalle de la cartografía de estas estructuras permitió además retirar algunos trazos de fallas que aparecían como inferidas en la cartografía existente.

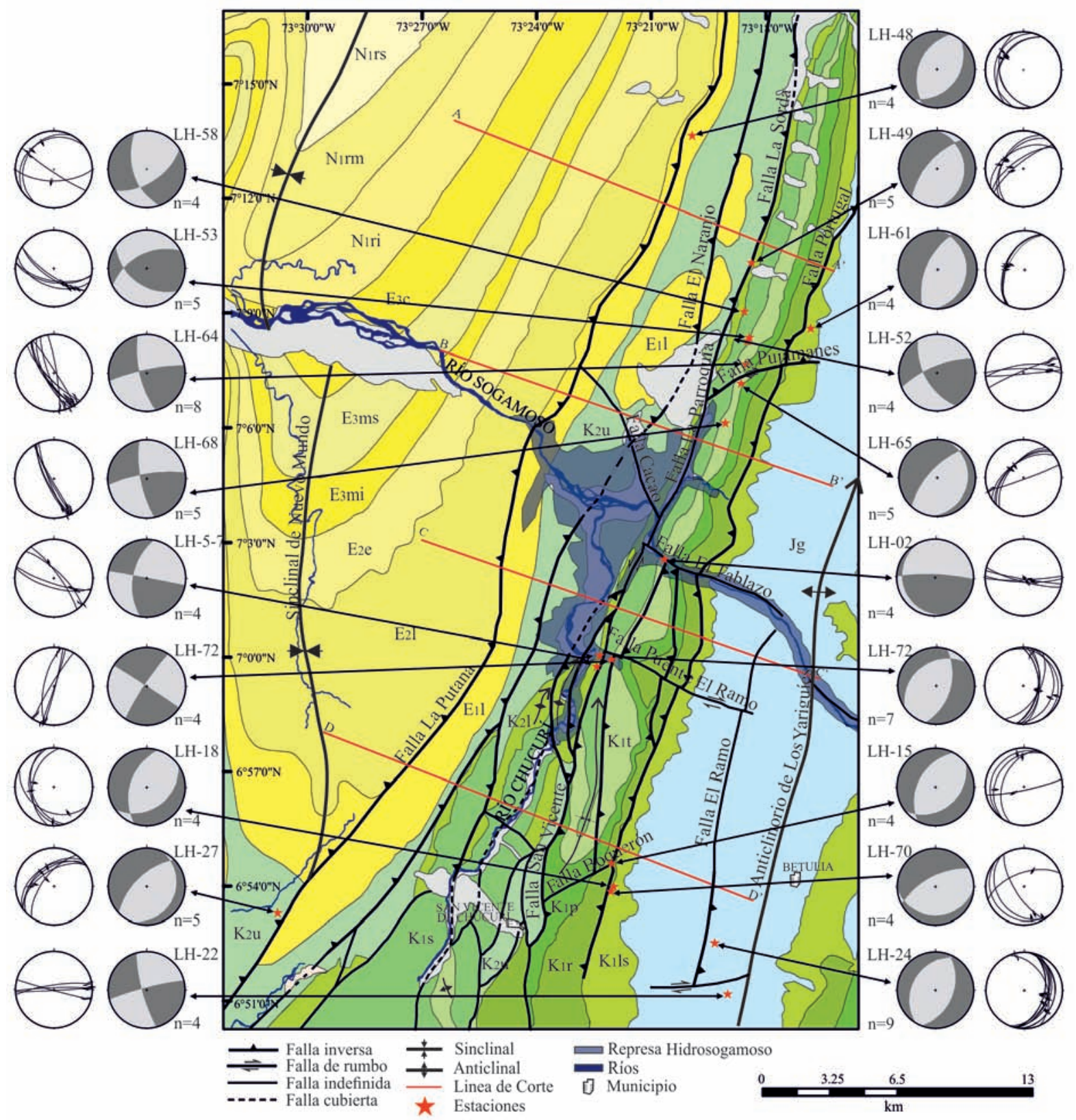

FIGURA 6. Mapa geológico mostrando la ubicación de las estaciones con datos de estrías asociados a la etapa tres, Gráficos de Angelier y Diagramas de Diedros Rectos NDA (balón de playa) correspondientes. 


\section{Estilo estructural}

La actualización cartográfica se llevó a cabo en las estructuras que afectan a las unidades del Cretácico y Paleógeno en la zona (FIGURAS 2 y 6). Se realizaron cuatro secciones transversales (FIGURA 7) a partir de esta cartografía geológica. Los modelos muestran estilos estructurales de cabalgamientos. También, el detalle cartográfico permitió definir el trazo de algunas fallas transversales, además de las estructuras longitudinales conocidas en la cordillera.

Los estilos estructurales identificados en la zona se presentan con características propias según el comportamiento mecánico diferencial entre los tipos de litología. En el caso de estudio, las rocas de la Formación Girón (unidad más antigua del área y que representa el núcleo del AY) es afectada por fallas con una geometría de mayor ángulo, asociadas con rampas frontales. Las formaciones geológicas cretácicas muestran fallas que involucran zonas llanas (flats) entre litologías blandas y zonas de rampa en rocas más competentes, característico de un estilo estructural de piel delgada. Además, la zona se ve afectada por fallas secundarias desarrolladas en pliegues, que a su vez están asociadas a fallas principales ubicadas más en profundidad.

La zona de estudio está dividida por fallas transversales que actúan como rampas laterales (FIGURA 8A) de las fallas longitudinales (FIGURAS 2 y 6). Estas fallas transversales segmentan la zona en tres bloques (sur, centro y norte) que se comportan de manera diferencial.
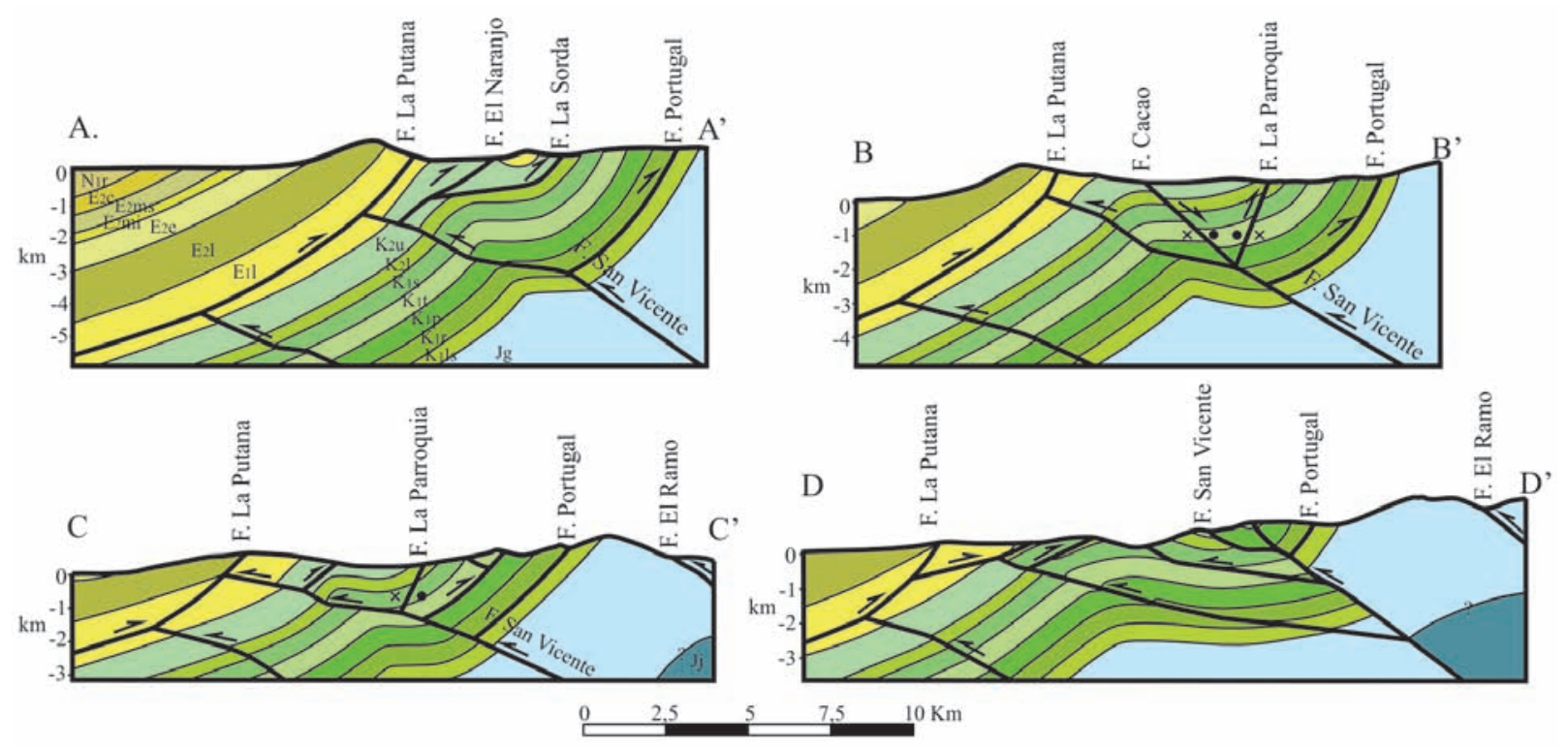

FIGURA 7. Cortes geológicos que abarcan la parte norte del AY (flanco oeste del SNM). A. Línea de corte A-A’. B. Línea de corte B-B'. C. Línea de corte C-C'. D. Línea de corte D-D'. Escala vertical igual a la horizontal. Para localización de los perfiles ver FIGURAS 2 y 6.

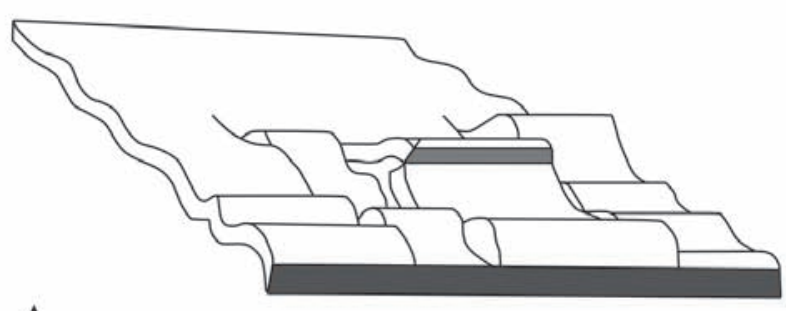

A.

FIGURA 8. A. Modelo de pliegues menores asociados al flanco del anticlinal y relacionado con fallas transversales como rampas laterales. Esquema tomado de Davis y Reynolds (1996). B. Esquema explicativo de generación de fallas secundarias a partir del núcleo de un sinclinal y hacia afuera de éste, el cual se infiere para la Falla La Putana en el flanco oriental del SNM (occidental del AY). Tomado de Mitra (2002). 
El bloque norte es afectado en profundidad por la Falla San Vicente y cerca de la superficie por fallas que presentan características de propagación mixta, ya que éstas son derivadas de la falla principal, pero su comportamiento es influenciado por el desarrollo de los pliegues menores, actuando como fallas secundarias (FIGURAS 7A y 8B). El bloque centro está más levantado estructuralmente respecto al bloque norte y está limitado por la Falla Pujamanes (FIGURAS 2 y 6). Este bloque evidencia mejor la transcurrencia presente en la zona, pues de la Falla San Vicente se desprende un retro-cabalgamiento que además presenta un movimiento sinestral (FIGURAS 7B y 7C). El bloque sur, cuyo límite con el bloque centro es la Falla Puente El Ramo (FIGURAS 2 y 6), se encuentra más levantado que los anteriores y es afectado por la Falla San Vicente (FIGURA 7D). La anterior observación acerca del levantamiento relativo del bloque sur es soportada también por la zona de cabeceo del SNM, donde sólo se encuentran las formaciones Lisama (Paleoceno) y La Paz (Eoceno) (FIGURAS 2 y 6). Este bloque presenta un estilo de fallamiento de las unidades cretácicas caracterizado por llanas, rampas y retro-cabalgamientos asociados a la Falla San Vicente, con vergencia general al occidente (WNW). Adicionalmente, la Falla La Putana se desprende de una falla a profundidad, pero el desarrollo de la deformación dentro del SNM permite su propagación como una falla secundaria hacia afuera del núcleo (FIGURAS 7D y 8B), afectando el flanco oriental del sinclinal en toda su extensión dentro del área de interés (FIGURAS 2 y 6).

Se resaltan las distintas vergencias que presentan las fallas longitudinales del flanco occidental del anticlinorio: mientras en el bloque sur la Falla San Vicente tiene una dirección de transporte tectónico al occidente, en el bloque norte se presenta un trazo con vergencia al oriente denominado Falla La Sorda (FIGURAS 2, 6 y 7). De igual manera, fallas longitudinales paralelas, como El Naranjo y Portugal, tienen vergencia al oriente. Este cambio de polaridad se presenta en el sector donde se localizan las fallas transversales de Puente El Ramo y El Tablazo, que además coincide con un control del curso del río Sogamoso (FIGURAS 2 y 6), sugiriendo una zona de transferencia, cuya temporalidad está por determinarse.

\section{Paleotensores registrados en las formaciones geológicas}

A partir del método aplicado se determinaron al menos tres tensores distintos de deformación asociados a las etapas propuestas para la evolución de la zona.
La etapa uno representa una fase de distensión y transcurrencia a final del Mesozoico, la segunda etapa se asocia al inicio de la deformación por compresión y la tercera etapa refleja la fase reciente responsable de la configuración actual. Cabe resaltar que estos tensores se encontraron en las diferentes unidades geológicas que presentan planos estriados (FIGURA 9) y por lo tanto, reflejan estas etapas, pero no excluyen otras posibles fases de deformación.

Los resultados de los tensores se presentan en la FIGURA 9 con el total de los datos analizados, incluyendo los medidos en zonas de transferencia. En primer lugar se procesaron los datos de planos de fallas según su tipo: normales (régimen distensivo), rumbo (régimen transcurrente) e inversas (régimen compresivo). Los diedros rectos por el método NDA indican el régimen predominante (en los casos en que se tuvieron al menos cuatro datos de planos estriados para procesar) y direcciones de máximo acortamientoextensión por el método P-T.

Un análisis adicional de los datos se puede encontrar en Cetina y Patiño (2013), donde se realizó el procesamiento de los datos sin incluir las estaciones con fallas transversales debido a que estas zonas pueden representar tensores locales e influenciar en los regionales. Sin embargo, la diferencia de los resultados fue mínima por lo que se decide no incluir este análisis en el presente artículo. Se describe entonces la evolución de los tensores de deformación con base en la totalidad de los datos habilitados en la fase de pre-procesamiento.

\section{Interpretación de la evolución de los tensores de deformación}

Las tres etapas de deformación identificadas para el flanco occidental del AY son representativas para el sector NW de la Cordillera Oriental de Colombia. Cada etapa evolutiva está asociada a unas condiciones tectónicas determinadas, responsables del origen y desarrollo de las estructuras.

\section{Etapa uno}

Esta primera etapa representa una fase distensiva transtensiva y fue identificada por los planos de falla que al rotarse a una estratificación de $0^{\circ}$ a $10^{\circ}$ revelaron una población de fallas normales y algunas de rumbo. Las formaciones Rosablanca y La Luna son las que mejor reflejan esta etapa, con una importante componente transtensiva registrada en las rocas del Cretácico 
Tardío (FIGURA 9), según la ubicación estratigráfica de los datos procesados. En estas dos unidades se observa una extensión NW-SE que está definida en las fallas longitudinales con rumbo NE que se muestran en los balones de playa (FIGURA 9) y en los trazos cartografiados (FIGURAS 2 y 6). Mientras tanto, en la Formación Tablazo la extensión ocurre en sentido N-S, pero relacionada con fallas transversales como la Falla El Tablazo, lo que se confirma al examinar la ubicación de los datos en zonas de transferencia (FIGURAS 6 y 9). Estas zonas están caracterizadas por fallas de rumbo y normales propias de ambientes distensivos en cuencas sedimentarias (McClay y Ellis, 1987). Para determinar la dirección de máxima extensión, asociada al tensor de esfuerzo principal menor $\left(\sigma_{3}\right)$, se procesaron por un lado la totalidad de los datos hallados en esta etapa y por otro los datos alejados de las zonas de transferencia, resultando direcciones de extensión en azimut de $133^{\circ}$ y $126^{\circ}$, respectivamente (FIGURA 10). Estas direcciones se pueden generalizar a NW-SE, es decir, con un tensor de acortamiento SW-NE que significa un régimen transtensivo si se aplica en estructuras longitudinales sur-norte.

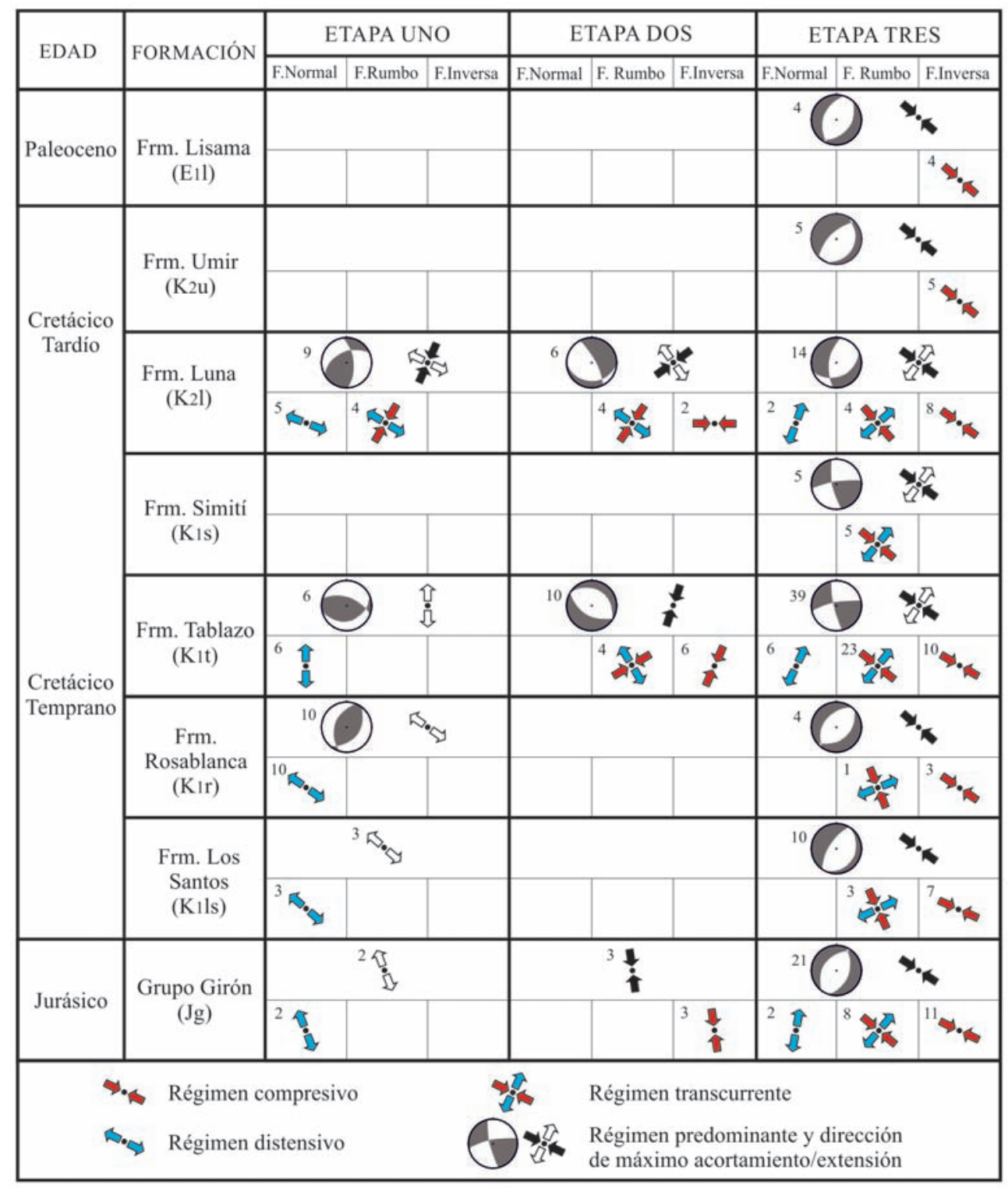

FIGURA 9. Representación del régimen tectónico predominante durante cada etapa de deformación en las unidades geológicas de la zona. El número que acompaña cada flecha indica la cantidad de datos de estrías utilizadas para el procesamiento. Ver mayor explicación en el texto. 


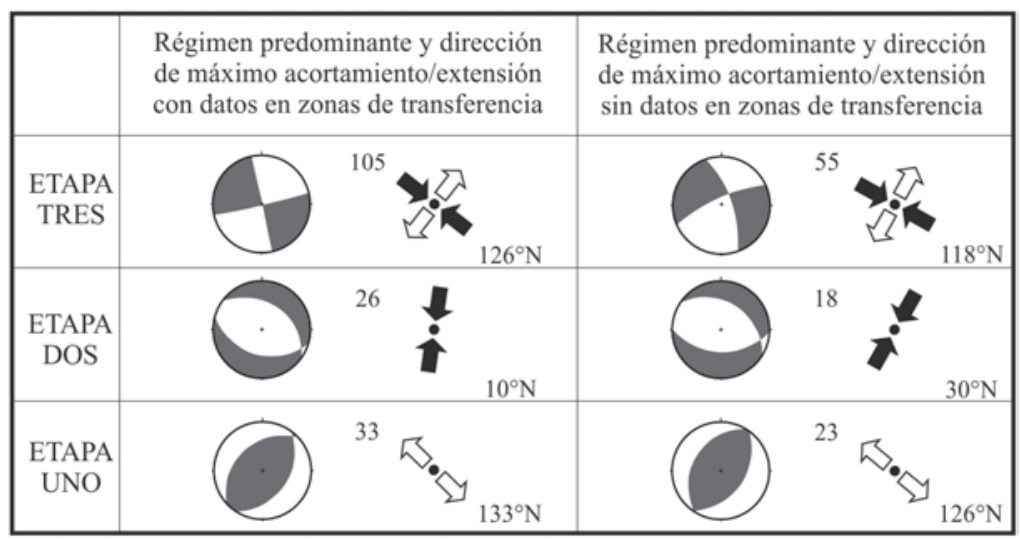

FIGURA 10. Tensores de deformación determinados para las tres etapas de plegamiento establecidas. Balón de playa generado con el método NDA y direcciones de máximo acortamiento-extensión (flechas negras y blancas, respectivamente) se obtienen con el método P-T. El número en la margen superior izquierda indica los datos de estrías procesados y en la margen inferior derecha la dirección del tensor en azimut.

\section{Etapa dos}

Esta etapa está relacionada con mayor acortamiento y configuración del flanco occidental del AY. Se refleja en datos obtenidos en las formaciones Girón, Tablazo y La Luna. Debido a la falta de datos este evento no se registra en las otras unidades cretácicas, aunque es muy probable encontrar su registro en áreas vecinas. La etapa dos es representada por planos de fallas inversas y de rumbo determinados mediante las rotaciones admitidas para esta fase. La dirección de máximo acortamiento obtenida en el procesamiento de todos los datos de esta etapa es de $10^{\circ}$ en azimut, mientras que la dirección obtenida sin procesar los datos de zonas de transferencia es $30^{\circ}$ en azimut (FIGURA 10), dirección más apropiada para asignarla como tensor regional (compresión NNE-SSW). Si se observan las direcciones obtenidas para las unidades de Girón, Tablazo y La Luna, se puede inferir una rotación progresiva en sentido horario del paleotensor de deformación, alcanzando una dirección E-W, observada en dos datos obtenidos en la Formación La Luna (FIGURA 9).

\section{Etapa tres}

Esta etapa se asocia a la fase deformativa responsable de la actual configuración estructural de la zona. Su determinación se realizó con datos de planos estriados no rotados, o levemente rotados, asumiendo que estos se generaron por la deformación reciente del anticlinorio, y revisando el cumplimiento de las consideraciones definidas en la metodología (TABLA 1). La etapa está representada de manera sistemática en las unidades geológicas y es registrada por conjuntos de planos de fallas inversas, de rumbo y algunas normales. Para determinar las direcciones de máximo acortamiento horizontal, al igual que en la etapa uno y dos, se optó por realizar dos procesamientos, uno con la totalidad de los datos que hacen parte de esta etapa y otro retirando aquellos datos tomados en zonas de transferencia. La dirección de máximo acortamiento obtenida procesando la totalidad de datos es de $126^{\circ}$ en azimut, mientras que el resultado del procesamiento discriminando las zonas de transferencia mostró una dirección de $118^{\circ}$ en azimut (FIGURA 10). En general se asume una dirección de acortamiento WNW-ESE, relacionada al actual tensor regional de deformación.

Para esta etapa se observa el predominio de acortamiento en las formaciones Girón, Los Santos y Rosablanca, así como una cinemática en rumbo en Tablazo, Simití y La Luna, y de nuevo acortamiento neto en las unidades más jóvenes del área (Umir y Lisama). Probablemente este cambio se debe a la orientación de los planos de falla medidos en cada unidad geológica, sobre todo si se involucran planos de falla medidos cerca a las estructuras transversales.

Finalmente, con el objetivo de observar la utilidad de la técnica de rotación en la determinación del actual tensor regional, se procesaron todos los datos de campo sin rotación $(\mathrm{n}=178)$, para analizarlos y comparar su resultado con el hallado para la etapa tres que representa el tensor actual $(\mathrm{n}=105)$. En la FIGURA 11 se observa que la variación en el tensor de deformación es mínima ( $5^{\circ}$ en azimut), lo que demuestra que al menos para áreas de baja complejidad estructural, la determinación de un tensor regional de deformación con datos "crudos" (sin rotar) es confiable si se compara el resultado con un procesamiento y análisis 
más detallado que considere fases de deformación como el realizado en este trabajo. Sin embargo, la metodología aplicada fue indispensable para separar paleo-tensores asociados con superposición de eventos de deformación durante la evolución de estructuras regionales como el AY.

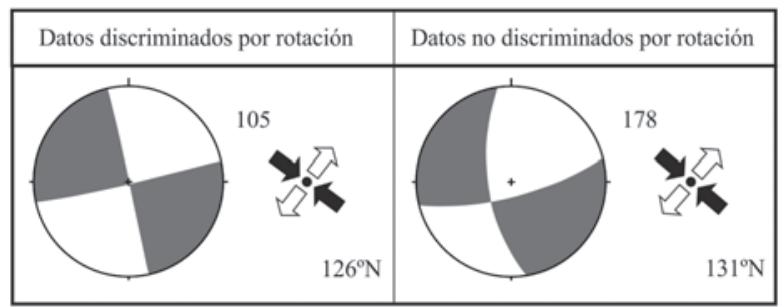

FIGURA 11. Tensores de deformación determinados con los datos discriminados por rotación (correspondientes a la etapa tres) y con todos los datos (sin rotación). En ambos casos resulta un tensor con fallas de rumbo que se muestra los gráficos de diedros rectos (por método NDA). Las direcciones de máximo acortamiento-extensión (flechas negras y flechas blancas, respectivamente) se obtienen con el método P-T. El número en la margen superior izquierda indica los datos de estrías procesados y en la margen inferior derecha la dirección del tensor en azimut.

\section{DISCUSIÓN}

Los resultados expuestos en el capítulo anterior se discuten a continuación enfocados principalmente en dos tópicos: el estilo estructural de la zona y los tensores de deformación involucrados.

El Anticlinorio de Los Yariguíes (AY) hace parte del bloque colgante de la Falla La Salina (FIGURA 1), una antigua estructura con historia de inversión tectónica que durante la etapa distensiva del Mesozoico limitó hacia el occidente una cuenca tipo graben (Sánchez et al., 2012). Según estos autores, por un posible arreglo estructural heredado (en echellon) se generaron otras estructuras longitudinales y paralelas al rumbo de las unidades geológicas, las cuales presentaron vergencia al occidente, tal como la Falla San Vicente, una falla profunda que afecta el basamento (Mojica y Franco, 1990). Esta estructura posee ramificaciones con igual vergencia y retro-cabalgamientos con vergencia al oriente, que posiblemente generan un sistema de dúplex de techos pasivos (Sánchez et al., 2012), un modelo que acogemos por lo observado en el presente trabajo (FIGURA 7). La Falla San Vicente se interpreta como responsable del levantamiento del AY, hipótesis soportada por Caballero et al. (2010) y Sánchez et al. (2012), quienes atribuyen este levantamiento a la propagación de fallas con vergencia hacia el occidente.
Modelos del subsuelo propuestos por Acosta (2002), López (2003) y Tesón et al. (2013) muestran también propagación de fallas profundas en el flanco occidental del anticlinorio, con vergencia al occidente.

Las fallas transversales corresponden probablemente a zonas de transferencia formadas durante el crecimiento de cuencas tipo rifting o back-arc en el Jurásico-Cretácico. Los datos obtenidos en la zona de estudio indican que estas fallas de rumbo, algunas con componente inverso, fueron reactivadas durante las etapas de compresión del Cenozoico y, por lo tanto, su movimiento actual está relacionado al tensor de deformación de la etapa tres, coexistiendo con las estructuras principales longitudinales que afectan la secuencia cretácica, las cuales son cortadas por estas fallas transversales, con un leve desplazamiento dextral en el flanco occidental del AY (FIGURAS 2 y 6). Estas estructuras transversales han sido documentadas en la deformación superficial relacionada con la reactivación de fallas de basamento (e.g. Mojica y Franco, 1990; Jiménez et al., 2016).

Las estructuras presentes en la zona se asocian a las tres etapas de desarrollo propuestas para la evolución del flanco occidental del AY y oriental del SNM. Estas etapas están implícitamente registradas en la estratigrafía de la cuenca del VMM, especialmente durante el Cenozoico. Específicamente, en el SNM se han realizado varios estudios (e.g. Caballero et al., 2010; Moreno et al., 2011; Nie et al., 2012), con el objetivo de determinar la fuente de sedimentos para las formaciones cenozoicas de la cuenca y sus implicaciones en la historia de deformación y exhumación de los orógenos adyacentes (cordilleras Oriental y Central). Con base en estos estudios y las etapas de deformación propuestas en el presente trabajo se hace una discusión sobre la evolución de esta parte de la CO.

La primera etapa de deformación propuesta para la zona se define como distensión-transtensión que afectó el área al final del Mesozoico, representada por antiguas fallas normales con una dirección de máxima extensión de $126^{\circ}$ en azimut (FIGURA 10). Las fallas debieron tener una orientación subparalela con la dirección de acreción de la Cordillera Occidental, la cual se ha reportado por varios autores en el Cretácico Tardío (e.g. Kerr et al., 1997; Villagómez et al., 2011). La primera etapa de deformación registrada aquí se podría asociar con el régimen tectónico provocado por el inicio de esta acreción. 
Esta convergencia oblicua progresivamente generó una dinámica transcurrente y cambio en los tensores de deformación y regímenes de esfuerzos (de distensivos-transtensivos a compresivos-transpresivos), configurándose la cuenca foreland pre-Andina (Cooper et al., 1995). En la Formación Lisama se registra el cambio en la fuente de sedimentos del cratón en el Paleoceno temprano, a la Cordillera Central en el Paleoceno tardío, lo que indica que la Cordillera Oriental aún no iniciaba su levantamiento (Moreno et al., 2011; Nie et al., 2012). Basados en estudios de termocronología de baja temperatura, trabajos de Parra et al. (2009), Duddy et al. (2009) y Restrepo-Moreno et al. (2019) asignan una edad del Paleoceno para el inicio de la exhumación de la zona cercana al AY. Lo anterior es acorde a un inicio de la deformación de la Cordillera Oriental mas no de su levantamiento, pues estudios de paleo-corrientes no referencian el AY como fuente de sedimentos, quizás porque no existía una topografía suficiente para generar una red de drenaje hacia el occidente (Caballero et al., 2010; RestrepoMoreno et al., 2019).

Este inicio de la deformación del AY es atribuido a la etapa dos del desarrollo de la estructura determinada en el presente trabajo, la cual presenta una dirección de máximo acortamiento de $30^{\circ}$ en azimut que puede atribuirse a la configuración tectónica del borde noroccidental de Suramérica durante el Paleoceno propuesta por Pindell y Kennan (2009), donde el tensor de deformación hallado se asemeja a las direcciones de convergencia de las placas para este tiempo, con convergencia oblicua de la Placa Farallón bajo la Placa Suramericana y movimiento de la Placa Caribe hacia el noreste.

Por otra parte, Cortés et al. (2005) determinan una evolución de paleo-esfuerzos asociados a la Cuenca del Valle Superior del Magdalena, en especial en el Sinclinal de Guaduas, hallando una dirección de esfuerzos E-W a WSW-ENE actuando desde el Maastrichtiano al Paleoceno tardío. Estos resultados se asemejan a las direcciones determinadas en el presente trabajo para la etapa dos, atribuida al Paleoceno y con las pocas variaciones en dirección que se deducen para la etapa uno, que actuaría desde el Maastrichtiano (FIGURA 9).

Posteriormente ocurre la discordancia del Eoceno medio, causada posiblemente por la etapa de máxima deformación de la Cordillera Central (Villamil, 1999;
Gómez et al., 2003; Restrepo-Moreno et al., 2019), por el cambio relativo de divergencia a convergencia entre las Américas (Cortés et al., 2005) y/o por el acople del Plateau Caribe bajo Suramérica (e.g. Villagómez et al., 2011). Durante el Eoceno medio al Oligoceno temprano se presenta la deposición de las formaciones La Paz y Esmeraldas, cuya fuente de sedimentos en general es atribuida a la Cordillera Central (e.g. Caballero et al., 2010). Estas formaciones presentan disminución de su espesor hacia el occidente del SNM debido al crecimiento del AY (Caballero et al., 2010). En el Oligoceno tardío y Mioceno temprano se depositan las formaciones Mugrosa y Colorado que registran como fuente de sedimentos a la Cordillera Oriental debido al levantamiento y erosión de la zona del AY (Caballero et al., 2010). Este levantamiento se atribuye al tensor de deformación determinado para la etapa tres, el cual se asocia a una dirección de máximo acortamiento de $118^{\circ}$ en azimut. Al igual que el tensor asignado a la etapa dos, el tensor de la etapa tres se asemeja al determinado por Cortés et al. (2005) en el Sinclinal de Guaduas, el cual varía de NW-SE a WNW-ESE desde el Eoceno hasta el Plioceno. Por otra parte, el tensor de deformación encontrado para esta etapa tres puede estar relacionado con la interacción de bloques oceánicos acrecionados al margen occidental de Suramérica desde el Eoceno hasta el Mioceno, en especial el Bloque Chocó-Panamá (Duque-Caro, 1990), también mencionado por Taboada et al. (2000) y Audemard y Audemard (2002), quienes relacionan esta acreción con una dirección de esfuerzos NW-SE y a una etapa andina temprana con exhumación de la Cordillera Oriental.

En general para la zona de estudio, con los resultados del presente trabajo (TABLA 7), se observa una progresión en la deformación desde el comienzo de la acreción de la Cordillera Occidental, inicialmente de carácter distensivo-transtensivo asociado a la etapa uno (con unidades no plegadas o levemente plegadas) y, posteriormente, de naturaleza compresiva-transpresiva, atribuido a las etapas dos y tres de deformación, cuyos tensores de acortamiento muestran una evolución en sentido horario. Finalmente, se asocia la transcurrencia (reflejada en los datos obtenidos durante la etapa tres) con la dinámica de estructuras locales heredadas de zonas de transferencia antiguas (deformación que induce esfuerzos) y no se contradice la hipótesis que sugiere un régimen de esfuerzos compresivo para la Cuenca del VMM hasta los $7^{\circ}$ en latitud norte (Tesón et al., 2013; Velandia, 2017). 
TABLA 7. Resumen de aspectos tectónicos y sedimentológicos relacionados con las etapas de deformación determinadas.

\begin{tabular}{|c|c|c|c|c|}
\hline $\begin{array}{c}\text { Tiempo } \\
\text { (Gómez et al., 2005) }\end{array}$ & Formación & Implicaciones & Etapa & $\begin{array}{l}\text { Evento } \\
\text { Tectónico }\end{array}$ \\
\hline $\begin{array}{l}\text { Mioceno temprano } \\
\text { Oligoceno tardío }\end{array}$ & $\begin{array}{l}\text { Colorado } \\
\text { Mugrosa }\end{array}$ & $\begin{array}{l}\text { Exhumación y erosión de la Cordillera Oriental } \\
\text { (Caballero et al., 2010) y por ende del AY }\end{array}$ & $\begin{array}{c}3 \\
\text { Dirección } \\
\text { de máximo } \\
\text { acortamiento: } \\
118^{\circ} \mathrm{N}\end{array}$ & $\begin{array}{c}\text { Acreción diacrónica de bloques } \\
\text { oceánicos } \\
\text { (Duque-Caro, 1990) }\end{array}$ \\
\hline $\begin{array}{l}\text { Oligoceno temprano } \\
\text { Eoceno medio }\end{array}$ & $\begin{array}{l}\text { Esmeralda } \\
\text { La Paz }\end{array}$ & $\begin{array}{l}\text { Disminución de espesores hacia el W del SNM } \\
\text { debido al crecimiento del AY (Caballero et al., } \\
\text { 2010) }\end{array}$ & & \\
\hline Eoceno medio & & Erosión generalizada & & $\begin{array}{l}\text { Máxima deformación en la } \\
\text { Cordillera Central (Villamil, } \\
\text { 1999; Gómez et al., 2003) }\end{array}$ \\
\hline Paleoceno tardío & Lisama & $\begin{array}{l}\text { Inicio de exhumación representativa en la } \\
\text { Cordillera Central y deformación temprana en } \\
\text { el área cercana al AY, sin representar fuente de } \\
\text { sedimentos (Parra et al., 2009; Duddy et al., } \\
\text { 2009; Restrepo-Moreno et al., 2019) }\end{array}$ & $\begin{array}{c}2 \\
\text { Dirección } \\
\text { de máximo } \\
\text { acortamiento: }\end{array}$ & $\begin{array}{l}\text { Movimiento de la Placa Caribe } \\
\text { hacia el NE. Convergencia } \\
\text { oblicua de la Placa Farallón } \\
\text { (e.g. Pindell y Kennan, 2009) }\end{array}$ \\
\hline \multicolumn{5}{|l|}{ Paleoceno temprano } \\
\hline Maastrichtiano & Umir & $\begin{array}{l}\text { Inicio de deformación en la Cordillera Central } \\
\text { (Gómez et al., 2005; Restrepo-Moreno et al., } \\
\text { 2019) }\end{array}$ & $\begin{array}{c}1 \\
\text { Dirección } \\
\text { de máxima } \\
\text { extensión: } 126^{\circ} \mathrm{N}\end{array}$ & $\begin{array}{l}\text { Acreción de la Cordillera } \\
\text { Occidental (Kerr et al., 1997) }\end{array}$ \\
\hline
\end{tabular}

\section{CONCLUSIONES}

El flanco occidental del AY, donde afloran las unidades geológicas que caracterizan la estratigrafía de la cuenca del Valle Medio del Magdalena, presenta un estilo estructural de fallas de cabalgamiento de alto a bajo ángulo. Éste se refleja principalmente en la Falla San Vicente, la cual es una estructura profunda con posible historial de inversión tectónica, que afecta las rocas más antiguas de la zona y cuya propagación en litologías cretácicas produce una serie de ramificaciones de fallas inversas con geometrías de llanas y rampas en cabalgamientos y retro-cabalgamientos. La anterior dinámica permite la generación de pliegues menores a lo largo del flanco occidental del AY, separados por fallas transversales que actúan como rampas laterales.

Se estableció un método de análisis estructural aplicado a zonas de baja complejidad estructural (a nivel regional) para el análisis y tratamiento de datos poblacionales de estrías de falla y evolución de pliegues (actitud de los estratos) con el objetivo de definir tensores de deformación. Se plantea un análisis puntual preliminar de los datos de estrías para determinar si presentan o no las óptimas condiciones mecánicas para su generación, según bases teóricas y presunciones geológicas del área. En datos que no cumplieron con las presunciones mecánicas se utilizó la técnica de rotación de planos de falla respecto a la actitud de las capas, esto para reconstruir condiciones previas y para determinar superposición de eventos. A partir de este método se determinaron tensores de deformación antiguos y actuales asociados a planos de fallas rotados y no rotados, respectivamente. Según el grado de rotación de las capas, los tensores de deformación resultantes se asocian con la evolución del flanco occidental del AY, representativa de la deformación al NW de la Cordillera Oriental de Colombia.

Se determinaron tensores relacionados a una progresión de la deformación con tres etapas observadas. La primera se asocia con una dirección de extensión (relacionada con el esfuerzo mínimo horizontal) en dirección $126^{\circ}$, correspondiente a un régimen distensivo-trantensivo que afectó la zona al final del Mesozoico. La segunda etapa presenta una orientación de máximo acortamiento (similar a un paleo-esfuerzo máximo horizontal) en dirección $30^{\circ}$, relacionada con un régimen compresivo-transpresivo en la zona, con mayor influencia durante el Paleoceno. Finalmente, la etapa tres muestra una dirección de acortamiento (similar a un esfuerzo máximo horizontal) en dirección $118^{\circ}$, relacionado a la última etapa de deformación andina, la cual tiene efecto desde el Oligoceno tardío hasta el presente. 


\section{AGRADECIMIENTOS}

Al Grupo de Investigación en Geología Básica y Aplicada (GIGBA) de la Escuela de Geología (UIS). Los autores agradecen la colaboración de ISAGEN y del Smithsonian Tropical Research Institute, en especial a Carlos Jaramillo y Edwin Cadena, por facilitar el acceso a las obras del proyecto Hidrosogamoso. Agradecemos también las observaciones y comentarios de los profesores Gustavo Hincapié Jaramillo y José Fernando Duque Trujillo, los cuales permitieron hacer aclaraciones en el texto y mejorar el manuscrito.

\section{REFERENCIAS}

Acosta, J. (2002). Estructura tectónica y modelos en 3D del Piedemonte Occidental de la Cordillera Oriental y del Valle Medio del Magdalena, Colombia. Publicaciones Especiales de INGEOMINAS.

Acosta, J., Velandia, F., Osorio, J., Lonergan, L., and Mora, H. (2007). Strike-slip deformation within the Colombian Andes. In: A.C. Ries, R.W.H. Butles, R.H. Graham (eds.). Deformation of the Continental Crust: The Legacy of Mike Coward (pp. 303-319). Geological Society of London, Special Publications, 272.

Alfaro, E., and Holz, M. (2014). Review of the chronostratigraphic charts in the Sinú-San Jacinto Basin based on new seismic stratigraphic interpretations. Journal of South American Earth Sciences, 56, 139-169. doi: 10.1016/j. jsames.2014.09.004.

Anderson, E.M. (1951). The dynamics of faulting and dyke formation with applications to Britain. $2^{\text {nd }}$ ed. Edinburgh, Scotland: Oliver and Boyd.

Angelier, J. (1984). Tectonic analysis of fault slip data sets. Journal Geophysical Research, 89(B7), 5835-5848.

Audemard, F.E., and Audemard, F.A. (2002). Structure of the Mérida Andes, Venezuela: relations with The South America-Caribbean geodynamic interaction. Tectonophysics, 345(1-4), 1-26. doi: 10.1016/S0040-1951(01)00218-9.

Babín, R.B., y Gómez, D. (2010). Problemas de geología estructural. 5. Rotaciones. Reduca (Geología) Serie Geología Estructural, 2(1), 57-73.
Bernal-Olaya, R, Mann, P., and Escalona, A. (2015). Cenozoic tectonostratigraphic evolution of the lower Magdalena Basin, Colombia: An example of an under- to overfilled Forearc Basin. In: C. Bartolini, P. Mann (eds.). Petroleum geology and potential of the Colombian Caribbean Margin (pp. 345-398). AAPG Memoir 108.

Butler, K., and Schamel, S. (1988). Structure along the eastern margin of the Central Cordillera, Upper Magdalena Valley, Colombia. Journal of South American Earth Sciences, 1(1), 109-120. doi: 10.1016/0895-9811(88)90019-3.

Burg, J.P. (2011). Structural geology and tectonics. Zürich: ETH Zürich and Universität Zürich.

Caballero, V., Mora, A., Quintero, I., Blanco, V., Parra, M., Rojas, L.E., López, C., Sánchez, N., Horton, B., Stockli, D., and Duddy, I. (2013). Tectonic controls on sedimentation in an intermontane hinterland basin adjacent to inversion structures: The Nuevo Mundo syncline, Middle Magdalena Valley, Colombia. In: M. Nemcok, A. Mora, J.W. Cosgrove (eds.). Thick-Skin-Dominated Orogens: From Initial Inversion to Full Accretion (pp. 315-342). Geological Society of London, Special Publications, 377.

Caballero, V., Parra, M., y Mora, A. (2010). Levantamiento de la Cordillera Oriental de Colombia durante el Eoceno tardío-Oligoceno temprano: proveniencia sedimentaria en el Sinclinal de Nuevo Mundo, cuenca Valle Medio del Magdalena. Boletín de Geología, 32(1), 4577.

Cediel, F., Shaw, R., and Cáceres, C. (2003). Tectonic assembly of the Northern Andean block. In: C. Bartolini, R.T. Buffler, J. Blickwede (eds.). The Circum-Gulf of Mexico and the Caribbean: Hydrocarbon habitats, basin formation, and plate tectonics (pp. 815-848). AAPG Memoir 79.

Cetina, L., y Patiño, H.A. (2013). Determinación de los tensores de esfuerzos asociados a la evolución del flanco oeste del Anticlinal de Los Cobardes, Santander. Tesis de pregrado, Universidad Industrial de Santander, Bucaramanga, Colombia.

Colleta, B, Hebrard, F., Letouzey, J., Werner, P., and Rudkiewicz, J. (1990). Tectonic Style and Crustal Structure of the Eastern Cordillera (Colombia) 
From a Balanced Cross-Section. In: J. Letouzey (ed.). Petroleum and Tectonic in Mobile Belts (pp. 81-100). Paris: Editions Technip.

Cooper, M.A., Addison, F.T., Alvarez, R., Coral, M., Graham, R.H., Hayward, A.B., Howe, S., Martínez, J., Naar, J., Peñas, R., Pulham, A., and Taborda, A. (1995). Basin development and tectonic history of the Llanos Basin, Eastern Cordillera, and Middle Magdalena Valley, Colombia. AAPG Bulletin, 79(10), 1421-1443.

Cortés, M., and Angelier, J. (2005). Current states of stress in the northern Andes as indicated by focal mechanisms of earthquakes. Tectonophysics, 403(1-4), 29-58. doi: 10.1016/j. tecto.2005.03.020.

Cortés, M., Angelier, J., and Colletta, B. (2005). Paleostress evolution of the northern Andes (Eastern Cordillera of Colombia): implications on plate kinematics of the South Caribbean region. Tectonics, 24, 1-27. doi: 10.1029/2003TC001551.

Davis, G., and Reynolds, S. (1996). Structural geology of rocks and regions. NewYork: John Wiley \& Sons, Inc.

Dengo, C.A., and Covey, M.C. (1993). Structure of the Eastern Cordillera of Colombia: implications for trap styles and regional tectonics. AAPG Bulletin, 77(8), 1315-1337. doi: 10.1306/BDFF8E7A1718-11D7-8645000102C1865D.

Doblas, M. (1998). Slickenside kinematic indicators. Tectonophysics, 295(1-2), 187-197. doi: 10.1016/ S0040-1951(98)00120-6.

Duddy, I.R., Parra, P., Mora, C.A., and Pimentel, C.A. (2009). AFTA apatite fission track analysis constraints on the Mesozoic to Quaternary thermal and tectonic evolution of the Middle Magdalena Basin and Santander Massif, Eastern Cordillera, Bucaramanga area, Colombia. $X$ Simposio Bolivariano-Exploración Petrolera en las Cuencas Subandinas, Cartagena, Colombia.

Duque-Caro, H. (1990). The Chocó Block in the northwestern corner of South America: Structural, tectonostratigraphic, and paleogeographic implications. Journal of South American Earth Sciences, 3(1), 71-84. doi: 10.1016/08959811(90)90019-W.
Egbue, O., Kellogg, J., Aguirre, H., and Torres, C. (2014). Evolution of the stress and strain fields in the Eastern Cordillera, Colombia. Journal of Structural Geology, 58, 8-21. doi: 10.1016/j. jsg.2013.10.004.

Ego, F., Sébrier, M., Lavenu, A., Yepes, H., and Egues, A. (1996). Quaternary state of stress in the northern Andes and the restraining bend model of the Ecuadorian Andes. Tectonophysics, 259(1-3), 101-116. doi: 10.1016/0040-1951(95)00075-5.

Etayo, F., y Rodríguez, G.I.(1985). Edad de la Formación Los Santos. Proyecto Cretácico. Publicaciones Geológicas Especiales INGEOMINAS, 16, 1-5.

Farris, D.W., Jaramillo, C., Bayona, G., RestrepoMoreno, S.A., Montes, C., Cardona, A, Mora, A., Speakman, R.J., Glascock, M.D., and Valencia, V. (2011). Fracturing of the Panamanian Isthmus during initial collision with South America. Geology, 39(11), 1007-1010. doi: 10.1130/G32237.1.

Fletcher, R.C., and Pollard, D.D. (1999). Can we understand structural and tectonic processes and their products without appeal to a complete mechanics?. Journal of Structural Geology, 21(8-9), 1071-1088. doi: 10.1016/S01918141(99)00056-5.

Fossen, H. (2010). Structural geology. Cambridge: Cambridge University Press.

Gómez, E., Jordan, T.E., Allmendinger, R., Hegarty, K., and Kelley, S. (2005). Syntectonic Cenozoic sedimentation in the northern middle Magdalena Valley Basin of Colombia and implications for exhumation of the Northern Andes. GSA Bulletin, 117(5-6), 547-569. doi: 10.1130/B25454.1.

Gómez, E., Jordan, T.E., Allmendinger, R.W., Hegarty, K., Kelley, S., and Heizler, M. (2003). Controls on architecture of the late Cretaceous to Cenozoic Southern Middle Magdalena Valley Basin, Colombia. GSA Bulletin, 115(2), 131-147. doi: 10.1130/0016-7606(2003)115<0131:COAOTL >2 .0.CO;2.

Gómez-Tapias, J., Guevara, A.N., Ramírez, N.M., Mejía, D.J., Avella, M.T., Ospina, J.S., y Penagos, M.M. (2007). Compiladores. Mapa geológico de Colombia, escala 1: 1.000.000. INGEOMINAS, Bogotá, Colombia. 
Hincapié-Gómez, S., Cardona, A., Jiménez, G., Monsalve, G., Ramírez-Hoyos, L., and Bayona, G. (2018). Paleomagnetic and gravimetrical reconnaissance of Cretaceous volcanic rocks from the Western Colombian Andes: Paleogeographic connections with the Caribbean Plate. Studia Geophysica et Geodaetica, 62(3), 485-511. doi: 10.1007/s11200-016-0678-y.

Horton, B.K., Saylor, J.E., Nie, J., Mora, A., Parra, M., Reyes-Harker, A., and Stockli, D.F. (2010). Linking sedimentation in the northern Andes to basement configuration, Mesozoic extension, and Cenozoic shortening: Evidence from detrital zircon U-Pb ages, Eastern Cordillera, Colombia. Geological Society of America Bulletin, 122(910), 1423-1442. doi: 10.1130/B30118.1.

Huang, Q., and Angelier, J. (1989). Inversion of field data in fault tectonics to obtain the regional stressII. Using conjugate fault sets within heterogeneous families for computing palaeostress axes. Geophysical Journal International, 96(1), 139149. doi: 10.1111/j.1365-246X.1989.tb05256.x.

Jiménez, G., López, O., Jaimes, L., y Umaña, R.M. (2016). Variaciones en el estilo estructural relacionado con anisotropías de basamento en el Valle Medio del Magdalena. Revista de la Academia Colombiana de Ciencias Exactas, Físicas y Naturales, 40(155), 312-319. doi: 10.18257/raccefyn.293.

Kammer, A. (1999). Observaciones acerca de un origen transpresivo de la Cordillera Oriental. Geología Colombiana, 24, 29-53.

Kellogg, J.N., Vega, V., Stallings, T.C., and Aiken, C.L. (1995). Tectonic development of Panama, Costa Rica, and the Colombian Andes: constraints from Global Positioning System geodetic studies and gravity. In: P. Mann (ed.). Geologic and tectonic development of the Caribbean Plate Boundary in Southern Central America (pp.75-90). Boulder: Geological Society of America Special Paper, 295.

Kerr, A.C., Marriner, G.F., Tarney, J., Nivia, A., Saunders, A.D., Thirlwall, M.F., and Sinton, C.W. (1997). Cretaceous Basaltic Terranes in western Columbia: elemental, chronological and $\mathrm{Sr}-\mathrm{Nd}$ isotopic constraints on petrogenesis. Journal of Petrology, 38(6), 677-702. doi: 10.1093/ petroj/38.6.677.

López, E. (2003). Upper crust models of Colombia. INGEOMINAS, Bogotá, Colombia.

López-Cardona, M.C. (2006). Análisis de deformación tectónica en el piedemonte de las cordilleras Central y Occidental Valle del Cauca, Colombia Contribuciones paleosísmicas. Tesis de Maestría, Universidad de EAFIT, Medellín, Colombia.

Marrett, R., and Allmendinger, R.W. (1990). Kinematic analysis of fault-slip data. Journal of Structural Geology, 12(8), 973-986. doi: 10.1016/01918141(90)90093-E.

Marrett, R., and Allmendinger, R.W. (1991). Estimates of strain due to brittle faulting: sampling of fault populations. Journal of Structural Geology, 13(6), 735-738. doi: 10.1016/0191-8141(91)90034-G.

Marrett, R., and Peacock, D.C.P. (1999). Strain and stress. Journal of Structural Geology, 21(8-9), 1057-1063. doi: 10.1016/S0191-8141(99)00020-6.

Martínez, J.R (2002). Geología estructural y dinámica global. Salamanca, Universidad de Salamanca.

McClay, K.R., and Ellis, P.G. (1987). Geometries of extensional fault systems developed in model experiments. Geology, 15(4), 341-344.

McCourt, W.J., Aspden, J.A., and Brook, M. (1984). New geological and geochronological data from the Colombian Andes: continental growth by multiple accretion. Journal of the Geological Society, 141(5), 831-845.

Mejía, E., Velandia, F., Zuluaga, C., López, J., and Cramer, T. (2012). Análisis estructural al noreste del Volcán Nevado del Ruíz, Colombia - aporte a la exploración geotérmica. Boletín de Geología, 34(1), 27-41.

Mitra, S. (2002). Fold-accommodation faults. AAPG Bulletin, 86(4), 671-693.

Mojica, J., y Franco, R. (1990). Estructura y evolución tectónica del Valle Medio y Superior del Magdalena, Colombia. Geología Colombiana, 17, 41-64. 
Montes, C., Cardona, A., Jaramillo, C., Pardo, A., Silva, J., Valencia, V., Ayala, C, Pérez-Angel, L.C., Rodriguez-Parra, L.A., Ramírez, V., and Niño, H. (2015). Middle Miocene closure of the Central American Seaway. Science, 348(6231), 226-229. doi: 10.1126/science.aaa2815.

Mora, A., Parra, M., Strecker, M.R., Kammer, A., Dimaté, C., and Rodríguez, F. (2006). Cenozoic contractional reactivation of Mesozoic extensional structures in the Eastern Cordillera of Colombia. Tectonics, 25(2), 1-19. doi: 10.1029/2005TC001854.

Mora, A., Reyes-Harker, A., Rodríguez, G., Tesón, E., Ramírez-Arias, J.C., Parra, M., Caballero, V., Mora, J.P., Quintero, I., Valencia, V., Ibáñez, M., Horton, B., and Stockli, D. (2013). Inversion tectonics under increasing rates of shortening and sedimentation: Cenozoic example from the Eastern Cordillera of Colombia. In: M. Nemcok, A. Mora, J.W. Cosgrove (eds.). Thick-SkinDominated Orogens: From Initial Inversion to Full Accretion (pp. 411-442). Geological Society, London, Special Publications, 377.

Morales, L.G., Podesta, D.J., Hatfield, W.C., Tanner, H., Jones, S.H., Barker, M.H.S., O’Donoghue, D.J., Mohler, C.E., Dubois, E.P., Jacobs, C., and Goss, C.R. (1958). General geology and oil occurrences of Middle Magdalena Valley, Colombia. In: L.G. Weeks (ed.). Habitat of Oil Symposium (pp. 641695). Tulsa: American Association of Petroleum Geologists.

Mora-Páez, H., Kellogg, J.N., Freymueller, J.T., Mencin, D., Fernandes, R.M., Diederix, H., LaFemina, P., Cardona-Piedrahita, L., Lizarazo, S., Peláez-Gaviria, J.R., Díaz-Mila, F., BohórquezOrozco, O., Giraldo-Londoño, L., and CorchueloCuervo, Y. (2019). Crustal deformation in the northern Andes - A new GPS velocity field. Journal of South American Earth Sciences, 89, 76-91. doi: 10.1016/j.jsames.2018.11.002.

Moreno, C., Horton, B., Caballero, V., Mora, A., Parra, M., and Sierra, J. (2011). Depositional and provenance record of the Paleogene transition from foreland to hinterland basin evolution during Andean orogenesis, northern Middle Magdalena Valley Basin, Colombia. Journal of South American Earth Sciences, 32(3), 246-263. doi: 10.1016/j.jsames.2011.03.018.
Nie, J., Horton, B., Saylor, J., Mora, A., Mange, M., Garzione, C., Basu, A., Moreno, C., Caballero, V., and Parra, M. (2012). Integrated provenance analysis of a convergent retroarc foreland system: $\mathrm{U}-\mathrm{Pb}$ ages, heavy minerals, $\mathrm{Nd}$ isotopes, and sandstone compositions of the Middle Magdalena Valley basin, northern Andes, Colombia. EarthScience Reviews, 110(1-4), 111-126. doi: 10.1016/j.earscirev.2011.11.002.

Osorio-Afanador, D.A. (2016). Estratigrafía y deformación del Grupo Girón en el Anticlinorio de los Yariguíes (“Anticlinal de Los Cobardes”) sectores Zapatoca y Río Lebrija. Tesis de pregrado, Universidad Industrial de Santander, Bucaramanga, Colombia.

Parra, M., Mora, A., Sobel, E.R., Strecker, M.R., and González, R. (2009). Episodic orogenic front migration in the northern Andes: Constraints from low-temperature thermochronology in the Eastern Cordillera, Colombia. Tectonics, 28(4), 1-27. doi: 10.1029/2008TC002423.

Peacock, D.C.P., and Marrett, R. (2000). Strain and stress: Reply. Journal of Structural Geology, 22(9), 1369-1378. doi: 10.1016/S01918141(00)00047-X.

Petit, J.P. (1987). Criteria for the sense of movement on fault surfaces in brittle rocks. Journal of Structural Geology, 9(5-6), 597-608. doi: 10.1016/0191-8141(87)90145-3.

Pindell, J.L., and Kennan, L. (2009). Tectonic evolution of the Gulf of Mexico, Caribbean and northern South America in the mantle reference frame: an update. In: K.H James, M.A. Lorente, J.L. Pindell (eds.). The Origin and Evolution of the Caribbean Plate (pp. 1-55). Geological Society of London, Special Publications, 328.

Prieto, G.A., Beroza, G.C., Barrett, S.A., López, G.A., and Florez, M. (2012). Earthquake nests as natural laboratories for the study of intermediate-depth earthquake mechanics. Tectonophysics, 570-571, 42-56. doi: 10.1016/j.tecto.2012.07.019.

Reiter, F., and Acs, P. (1996-2014). TectonicsFP, Software for Structural Geology, Microsoft Windows. Innsbruck University, Austria. 
Restrepo-Moreno, S.A., Foster, D.A., Bernet, M., Min, K., and Noriega, S. (2019). Morphotectonic and Orogenic Development of the Northern Andes of Colombia: A Low-Temperature Thermochronology Perspective. In: F. Cediel, R.P. Shaw (eds.). Geology and Tectonics of Northwestern South America (pp. 749-832). Springer, Cham. doi: 10.1007/978-3-319-761329_11.

Rossello, E.A., and Cossey, S.P. (2012). What is the evidence for subduction in the Caribbean Margin of Colombia?. XI Simposio Bolivariano - Exploración Petrolera en las Cuencas Subandinas. Cartagena, Colombia.

Royero, J., y Clavijo, J. (2001). Mapa geológico generalizado Departamento de Santander. Escala 1:300.000. Memoria Explicativa. INGEOMINAS, Bogotá, Colombia.

Sánchez, J., Horton, B., Tesón, E., Mora, A., Ketcham, R., and Stockli, D. (2012). Kinematic evolution of Andean fold-thrust structures along the boundary between the Eastern Cordillera and Middle Magdalena Valley basin, Colombia. Tectonics, 31(3), 1-24. doi: 10.1029/2011TC003089.

Sarmiento-Rojas, L.F. (2001). Mesozoic rifting and Cenozoic basin inversion history of the Eastern Cordillera, Colombian Andes. Inferences from tectonic models. Ph.D. Thesis, Vrije Universiteit, Amsterdam, the Netherlands.

Sarmiento-Rojas, L.F., Van Wess, J., and Cloetingh, S. (2006). Mesozoic transtensional basin history of the Eastern Cordillera, Colombian Andes: Inferences from tectonic models. Journal of South American Earth Sciences, 21(4), 383-411. doi: 10.1016/j.jsames.2006.07.003.

Taboada, A., Rivera, L.A., Fuenzalida, A., Cisternas, A., Philip, H., Bijwaard, H., Olaya, J., and Rivera, C. (2000). Geodynamics of the northern Andes: subductions and intracontinental deformation (Colombia). Tectonics, 19(5), 787-813. doi: 10.1029/2000TC900004.

Tesón, E., Mora, A., Silva, A., Namson, J., Teixell, A., Castellanos, J., Casallas, W., Julivert, M., Taylor, M., Ibáñez, M., and Valencia, V. (2013). Relationship of Mesozoic graben development, stress, shortening magnitude, and structural style in the Eastern Cordillera of the Colombian Andes. In: M. Nemcok, A. Mora, J.W. Cosgrove (eds.). Thick-Skin-Dominated Orogens: From initial inversion to full accretion (pp. 257-283). Geological Society, London, Special Publications, 377.

Toto, E.A., and Kellogg, J.N. (1992). Structure of the Sinu-San Jacinto fold belt - an active accretionary prism in northern Colombia. Journal of South American Earth Sciences, 5(2), 211-222. doi: 10.1016/0895-9811(92)90039-2.

Trenkamp, R., Kellogg, J., Freymueller, J., and Mora, H. (2002). Wide plate margin deformation, southern Central America and northwestern South America, CASA GPS observations. Journal of South American Earth Sciences, 15(2), 157-171. doi: 10.1016/S0895-9811(02)00018-4.

Turner, F.J. (1953). Nature and dynamic interpretation of deformation lamellae in calcite of three marbles. American Journal of Science, 251(4), 276-298. doi: 10.2475/ajs.251.4.276.

Velandia, F. (2017). Cinemática de las fallas mayores del Macizo de Santander - énfasis en el modelo estructural y temporalidad al sur de la Falla de Bucaramanga. PhD Tesis. Universidad Nacional de Colombia, Bogotá, Colombia.

Velandia, F., and Bermúdez, M. (2018). The transpressive southern termination of the Bucaramanga fault (Colombia): Insights from geological mapping, stress tensors, and fractal analysis. Journal of Structural Geology, 115, 190207. doi: 10.1016/j.jsg.2018.07.020.

Velandia, F., Acosta, J., Terraza. R., and Villegas, H. (2005). The current tectonic motion of the Northern Andes along the Algeciras Fault System in SW Colombia. Tectonophysics, 399(1-4), 313329. doi: 10.1016/j.tecto.2004.12.028.

Velandia, F., Cetina, M.A., Castellanos, L.E. y Gómez, S. (2016). Análisis de fracturas y cinemática de fallas geológicas como primer aporte al modelo conceptual de aguas subterráneas en la zona de Charta, Macizo de Santander-Colombia. Revista de la Facultad de Ingeniería UCV, 31(2), 37-56.

Velásquez, R., y Castillo, V. (2006). Resistencia de la etnia Yareguíes a las políticas de reducción y 
"civilización" en el siglo XIX. Revista Historia y Sociedad, 12, 285-317.

Villagómez, D., Spikings, R., Magna, T., Kammer, A., Winkler, W., and Beltrán, A. (2011). Geochronology, geochemistry and tectonic evolution of the Western and Central cordilleras of Colombia. Lithos, 125(3-4), 875-896. doi: 10.1016/j.lithos.2011.05.003.

Villamil, T. (1999). Campanian-Miocene tectonostratigraphy, depocenter evolution and basin development of Colombia and western Venezuela. Paleogeography, Paleoclimatology, Paleoecology, 153(1-4), 239 -275. doi: 10.1016/ S0031-0182(99)00075-9.

Ward, D., Goldsmith, R., Cruz, J., y Restrepo, H. (1973). Geología de los cuadrángulos H-12
Bucaramanga y H-13 Pamplona, Departamento de Santander. Boletín Geológico, 21(1-3), 1-132.

Ward, W., Goldsmith, R., Jimeno, A., Cruz, J., Restrepo, H., y Gómez, E. (1977). Мара geológico del cuadrángulo H-12 Bucaramanga escala 1:100.000. INGEOMINAS.

\begin{tabular}{c}
\hline \hline Lina María Cetina-Tarazona. \\
ORCID: 0000-0001-6814-480X \\
Francisco Velandia. \\
ORCID: 0000-0001-6263-0903 \\
Hugo Alonso Patiño-Sanabria. \\
ORCID: 0000-0002-6210-226X \\
\hline \hline
\end{tabular}

Trabajo recibido: enero 03 de 2019

Trabajo aceptado: junio 10 de 2019 